\title{
Selection Games on Continuous Functions
}

\author{
Christopher Caruvana and Jared Holshouser
}

February 4, 2021

\begin{abstract}
In this paper we study the selection principle of closed discrete selection, first researched by Tkachuk in [13] and strengthened by Clontz, Holshouser in [3], in set-open topologies on the space of continuous real-valued functions. Adapting the techniques involving point-picking games on $X$ and $C_{p}(X)$, the current authors showed similar equivalences in [1] involving the compact subsets of $X$ and $C_{k}(X)$. By pursuing a bitopological setting, we have touched upon a unifying framework which involves three basic techniques: general game duality via reflections (Clontz), general game equivalence via topological connections, and strengthening of strategies (Pawlikowski and Tkachuk). Moreover, we develop a framework which identifies topological notions to match with generalized versions of the point-open game.
\end{abstract}

\section{Introduction}

The closed discrete selection principle was first studied by Tkachuk in 2017. This property occurs naturally in the course of studying functional analysis. Tkachuk connected this selection principle on $C_{p}(X)$ with topological properties of $X$. He then went on to consider the corresponding selection game, creating a partial characterization of winning strategies in that game and finding connections between it, the point-open game on $X$, and Gruenhage's $W$-game on $C_{p}(X)$ [14]. In 2019, Clontz and Holshouser [3] finished this characterization, showing that the discrete selection game on $C_{p}(X)$ is equivalent to a modification of the point-open game on $X$. Clontz and Holshouser show this not only for full information strategies but also for limited information strategies.

The current authors continued this work, researching the closed discrete game on $C_{k}(X)$, the real-valued continuous functions with the compact open topology [1]. They show that similar connections exist in this setting, with the point-open game on $X$ replaced by the compact-open game. They also isolated general techniques which have use beyond the study of closed discrete selections.

In this paper, we study the problem of closed discrete selection in the general setting of setopen topologies on the space of continuous functions. We use closed discrete selection as a tool not only for comparing $X$ to its space of continuous functions, but also for comparing different set-open topologies to each other. To establish these connections, we prove general statements in three categories:

1. strengthening the strategies in games,

2. criteria for games to be dual,

3. characterizations of strong strategies in abstract point-open games,

and use work of Clontz [2] to show that some general classes of games are equivalent.

In this version, we have 
- identified that Lemmas 7 and 8, as stated, are not known to be true yet and reference arXiv:2102.00296 for a revised proof for $k$-covers.

- corrected a slight error in the statement of Theorem 26 which relied on Lemma 8.

\section{Definitions and Preliminaries}

Definition 1. Let $X$ be a space and $\mathcal{A} \subseteq \wp(X)$. We say that $\mathcal{A}$ is an ideal-base if, for $A_{1}, A_{2} \in \mathcal{A}$, there exists $A_{3} \in \mathcal{A}$ so that $A_{1} \cup A_{2} \subseteq A_{3}$.

Definition 2. For a topological space $X$ and a collection $\mathcal{A} \subseteq \wp(X)$, we let $\overline{\mathcal{A}}=\left\{\operatorname{cl}_{X}(A): A \in \mathcal{A}\right\}$.

Definition 3. Fix a topological space $X$ and a collection $\mathcal{A} \subseteq \wp(X)$. Then

- we let $C_{p}(X)$ denote the set of all continuous functions $X \rightarrow \mathbb{R}$ endowed with the topology of point-wise convergence; we also let $\mathbf{0}$ be the function which identically zero.

- we let $C_{k}(X)$ denote the set of all continuous functions $X \rightarrow \mathbb{R}$ endowed with the topology of uniform convergence on compact subsets of $X$; we will write

$$
[f ; K, \varepsilon]=\left\{g \in C_{k}(X): \sup \{|f(x)-g(x)|: x \in K\}<\varepsilon\right\}
$$

for $f \in C_{k}(X), K \subseteq X$ compact, and $\varepsilon>0$,

- in general, we let $C_{\mathcal{A}}(X)$ denote the set of all continuous functions $X \rightarrow \mathbb{R}$ endowed with the $\mathcal{A}$-open topology; we will write

$$
[f ; A, \varepsilon]=\left\{g \in C_{\mathcal{A}}(X): \sup \{|f(x)-g(x)|: x \in A\}<\varepsilon\right\}
$$

for $f \in C_{\mathcal{A}}(X), A \in \mathcal{A}$, and $\varepsilon>0$,

Notice that, for the sets of the form $[f ; A, \varepsilon]$ to be a base for the topology $C_{\mathcal{A}}(X)$, then $\mathcal{A}$ must be an ideal-base.

Definition 4. For a topological space $X$, we let $K(X)$ denote the family of all non-empty compact subsets of $X$.

Definition 5. Let $X$ be a topological space. We say that $A \subseteq X$ is $\mathbb{R}$-bounded if, for every continuous $f: X \rightarrow \mathbb{R}, f[A]$ is bounded.

In this paper, we will be concerned with selection principles and related games. For classical results, basic tools, and notation, the authors recommend [10] and [7].

Definition 6. Consider collections $\mathcal{A}$ and $\mathcal{B}$ and an ordinal $\alpha$. The corresponding selection principles are defined as follows:

- $S_{\text {fin }}^{\alpha}(\mathcal{A}, \mathcal{B})$ is the assertion that, given any $\left\{A_{\xi}: \xi \in \alpha\right\} \subseteq \mathcal{A}$, there exists $\left\{\mathcal{F}_{\xi}: \xi \in \alpha\right\}$ so that, for each $\xi \in \alpha, \mathcal{F}_{\xi}$ is a finite subset of $A_{\xi}$ (denoted as $\mathcal{F}_{\xi} \in\left[A_{\xi}\right]^{<\omega}$ hereinafter) and $\bigcup\left\{\mathcal{F}_{\xi}: \xi \in \alpha\right\} \in \mathcal{B}$, and

- $S_{1}^{\alpha}(\mathcal{A}, \mathcal{B})$ is the assertion that, given any $\left\{A_{\xi}: \xi \in \alpha\right\} \subseteq \mathcal{A}$, there exists $\left\{x_{\xi}: \xi \in \alpha\right\}$ so that, for each $\xi \in \alpha, x_{\xi} \in A_{\xi}$ and $\left\{x_{\xi}: \xi \in \alpha\right\} \in \mathcal{B}$.

We suppress the superscript when $\alpha=\omega$; i.e., $S_{1}(\mathcal{A}, \mathcal{B})=S_{1}^{\omega}(\mathcal{A}, \mathcal{B})$. 
Definition 7. Let $X$ be a topological space and $\mathscr{U}$ be an open cover of $X$ with $X \notin \mathscr{U}$. Recall that

- $\mathscr{U}$ is said to be a $\Lambda$-cover if, for every $x \in X,\{U \in \mathscr{U}: x \in U\}$ is infinite,

- $\mathscr{U}$ is an $\omega$-cover of $X$ provided that given any finite subset $F$ of $X$, there exists some $U \in \mathscr{U}$ so that $F \subseteq U$

- $\mathscr{U}$ is said to be a $\gamma$-cover if $\mathscr{U}$ is an infinite $\omega$-cover and for every finite subset $F \subseteq X$, $\{U \in \mathscr{U}: F \nsubseteq U\}$ is finite,

- $\mathscr{U}$ is a $k$-cover of $X$ provided that given any compact subset $K$ of $X$, there exists some $U \in \mathscr{U}$ so that $K \subseteq U$, and

- $\mathscr{U}$ is said to be a $\gamma_{k}$-cover if $\mathscr{U}$ is an infinite $k$-cover and for every compact $K \subseteq X$, $\{U \in \mathscr{U}: K \nsubseteq U\}$ is finite.

Note that if $\mathscr{U}=\left\{U_{n}: n \in \omega\right\}$, then $\mathscr{U}$ is a $\gamma_{k}$-cover if and only if every cofinal sequence of the $U_{n}$ form an $k$-cover.

For a family of sets $\mathcal{A}$, let

- $\mathcal{O}(X, \mathcal{A})$ to be all open covers $\mathscr{U}$ so that $X \notin \mathscr{U}$ and for every $A \in \mathcal{A}$, there is an open set $U \in \mathscr{U}$ which contains $A$,

- $\Lambda(X, \mathcal{A})$ be all open covers $\mathscr{U}$ so that $X \notin \mathscr{U}$, and for all $A \in \mathcal{A}$, there are infinitely many $U \in \mathscr{U}$ so that $A \subseteq U$, and

- $\Gamma(X, \mathcal{A})$ to be all infinite open covers $\mathscr{U}$ so that $X \notin \mathscr{U}$ and for every $A \in \mathcal{A},\{U \in \mathscr{U}: A \nsubseteq$ $U\}$ is finite.

Remark 1. Note that

- $\mathcal{O}\left(X,[X]^{<\omega}\right)=\Omega_{X}$ denotes the collection of all $\omega$-covers of $X$.

- $\mathcal{O}(X, K(X))=\mathcal{K}_{X}$ denotes the collection of all $k$-covers of $X$.

- $\Gamma(X, K(X))=\Gamma_{k}(X)$ denotes the collection of all $\gamma_{k}$-covers of $X$.

Notation. We let

- For any collection $\mathcal{A}, \neg \mathcal{A}$ is the complement of $\mathcal{A}$.

- $\mathscr{T}_{X}$ denote the set of all non-empty subsets of $X$.

- $\Omega_{X, x}$ denote the set of all $A \subseteq X$ with $x \in \operatorname{cl}_{X}(A)$. We also call $A \in \Omega_{X, x}$ a blade of $x$.

- $\Gamma_{X, x}$ denote the set of all sequences $\left\{x_{n}: n \in \omega\right\} \subseteq X$ with $x_{n} \rightarrow x$.

- $\mathcal{D}_{X}$ denote the collection of all dense subsets of $X$.

- $\mathrm{CD}_{X}$ denote the collection of all closed and discrete subsets of $X$.

- $\mathcal{O}_{X}$ denote the collection of all open covers of $X$.

- $\Lambda_{X}$ denote the collection of all $\lambda$-covers of $X$. 
- $\Gamma_{X}$ denote the collection of all $\gamma$-covers of $X$.

We can create variations of selection principles and their negations by looking at selection games.

Definition 8. Given a set $\mathcal{A}$ and another set $\mathcal{B}$, we define the finite selection game $G_{\text {fin }}^{\alpha}(\mathcal{A}, \mathcal{B})$ for $\mathcal{A}$ and $\mathcal{B}$ as follows:

$$
\begin{array}{l|llllll}
\mathrm{I} & A_{0} & A_{1} & A_{2} & \cdots & A_{\xi} & \cdots \\
\hline \mathrm{II} & \mathcal{F}_{0} & \mathcal{F}_{1} & \mathcal{F}_{2} & \cdots & \mathcal{F}_{\xi} & \cdots
\end{array}
$$

where $A_{\xi} \in \mathcal{A}$ and $\mathcal{F}_{\xi} \in\left[A_{\xi}\right]^{<\omega}$ for all $\xi<\alpha$. We declare Two the winner if $\bigcup\left\{\mathcal{F}_{\xi}: \xi<\alpha\right\} \in \mathcal{B}$. Otherwise, One wins. We let $G_{\text {fin }}(\mathcal{A}, \mathcal{B})$ denote $G_{\text {fin }}^{\omega}(\mathcal{A}, \mathcal{B})$.

Definition 9. Similarly, we define the single selection game $G_{1}^{\alpha}(\mathcal{A}, \mathcal{B})$ as follows:

\begin{tabular}{c|cccccc}
$\mathrm{I}$ & $A_{0}$ & $A_{1}$ & $A_{2}$ & $\cdots$ & $A_{\xi}$ & $\cdots$ \\
\hline $\mathrm{II}$ & $x_{0}$ & $x_{1}$ & $x_{2}$ & $\cdots$ & $x_{\xi}$ & $\cdots$
\end{tabular}

where each $A_{\xi} \in \mathcal{A}$ and $x_{\xi} \in A_{\xi}$. We declare Two the winner if $\left\{x_{\xi}: \xi \in \alpha\right\} \in \mathcal{B}$. Otherwise, One wins. We let $G_{1}(\mathcal{A}, \mathcal{B})$ denote $G_{1}^{\omega}(\mathcal{A}, \mathcal{B})$.

Definition 10. We define strategies of various strength below.

- A strategy for player One in $G_{1}^{\alpha}(\mathcal{A}, \mathcal{B})$ is a function $\sigma:(\bigcup \mathcal{A})^{<\alpha} \rightarrow \mathcal{A}$. A strategy $\sigma$ for One is called winning if whenever $x_{\xi} \in \sigma\left\langle x_{\zeta}: \zeta<\xi\right\rangle$ for all $\xi<\alpha,\left\{x_{\xi}: \xi \in \alpha\right\} \notin \mathcal{B}$. If player One has a winning strategy, we write $\mathrm{I} \uparrow G_{1}^{\alpha}(\mathcal{A}, \mathcal{B})$.

- A strategy for player Two in $G_{1}^{\alpha}(\mathcal{A}, \mathcal{B})$ is a function $\tau: \mathcal{A}^{<\alpha} \rightarrow \cup \mathcal{A}$. A strategy $\tau$ for Two is winning if whenever $A_{\xi} \in \mathcal{A}$ for all $\xi<\alpha,\left\{\tau\left(A_{0}, \cdots, A_{\xi}\right): \xi<\alpha\right\} \in \mathcal{B}$. If player Two has a winning strategy, we write II $\uparrow G_{1}^{\alpha}(\mathcal{A}, \mathcal{B})$.

- A predetermined strategy for One is a strategy which only considers the current turn number. We call this kind of strategy predetermined because One is not reacting to Two's moves, they are just running through a pre-planned script. Formally it is a function $\sigma: \alpha \rightarrow \mathcal{A}$. If One has a winning predetermined strategy, we write $\mathrm{I} \underset{\text { pre }}{\uparrow} G_{1}^{\alpha}(\mathcal{A}, \mathcal{B})$.

- A Markov strategy for Two is a strategy which only considers the most recent move of player One and the current turn number. Formally it is a function $\tau: \mathcal{A} \times \alpha \rightarrow \bigcup \mathcal{A}$. If Two has a winning Markov strategy, we write II $\underset{\text { mark }}{\uparrow} G_{1}^{\alpha}(\mathcal{A}, \mathcal{B})$.

Definition 11. Two games $\mathcal{G}_{1}$ and $\mathcal{G}_{2}$ are said to be strategically dual provided that the following two hold:

- I $\uparrow \mathcal{G}_{1}$ iff II $\uparrow \mathcal{G}_{2}$

- I $\uparrow \mathcal{G}_{2}$ iff II $\uparrow \mathcal{G}_{1}$

Two games $\mathcal{G}_{1}$ and $\mathcal{G}_{2}$ are said to be Markov dual provided that the following two hold:

- I $\underset{\text { pre }}{\uparrow} \mathcal{G}_{1}$ iff II $\underset{\text { mark }}{\uparrow} \mathcal{G}_{2}$

- I $\underset{\text { pre }}{\uparrow} \mathcal{G}_{2}$ iff II $\underset{\text { mark }}{\uparrow} \mathcal{G}_{1}$ 
Two games $\mathcal{G}_{1}$ and $\mathcal{G}_{2}$ are said to be dual provided that they are both strategically dual and Markov dual.

Remark 2. In general, $S_{1}^{\alpha}(\mathcal{A}, \mathcal{B})$ holds if and only if I $\underset{\text { pre }}{\forall} G_{1}^{\alpha}(\mathcal{A}, \mathcal{B})$. See [3, Prop. 13].

Remark 3. The game $G_{\text {fin }}\left(\mathcal{O}_{X}, \mathcal{O}_{X}\right)$ is the well-known Menger game and the game $G_{1}\left(\mathcal{O}_{X}, \mathcal{O}_{X}\right)$ is the well-known Rothberger game.

Notation. For $A \subseteq X$, let $\mathscr{N}(A)$ be all open sets $U$ so that $A \subseteq U$. Set $\mathscr{N}[X]=\left\{\mathscr{N}_{x}: x \in X\right\}$, and in general if $\mathcal{A}$ is a collection of subsets of $X$, then $\mathscr{N}[\mathcal{A}]=\{\mathscr{N}(A): A \in \mathcal{A}\}$. In the case when $X$ and $X^{\prime}$ represent two topologies on the same underlying set, we will use the notation $\mathscr{N}_{X}(A)$ to denote the collection of open sets relative to the topology according to $X$ that contain $A$.

Remark 4. The game $G_{1}\left(\mathscr{N}[X], \neg \mathcal{O}_{X}\right)$ is the well-known point-open game first appearing in [4]: player One is trying to build an open cover and player Two is trying to avoid building an open cover. The game $G_{1}\left(\mathscr{N}[K(X)], \neg \mathcal{O}_{X}\right)$ is the compact-open game.

Generally, when $\mathscr{N}[\mathcal{A}]$ is being used in a game, we will use the identification of $A$ with $\mathscr{N}(A)$ to simplify notation. Particularly, One picks $A \in \mathcal{A}$ and Two's response will be an open set $U$ so that $A \subseteq U$.

Definition 12. A topological space $X$ is called discretely selective if, for any sequence $\left\{U_{n}\right.$ : $n \in \omega\}$ of non-empty open sets, there exists a closed discrete set $\left\{x_{n}: n \in \omega\right\} \subseteq X$ so that $x_{n} \in U_{n}$ for each $n \in \omega$; i.e. $S_{1}\left(\mathscr{T}_{X}, \mathrm{CD}_{X}\right)$ holds. This notion was first isolated by Tkachuk in [13].

Definition 13. For a topological space $X$, the closed discrete selection game on $X$, is $G_{1}\left(\mathscr{T}_{X}, \mathrm{CD}_{X}\right)$. Tkachuk studies this game in [14].

Note that $X$ is discretely selective if and only if $\underset{\text { I } \underset{\text { pre }}{\not}}{\not} G_{1}\left(\mathscr{T}_{X}, \mathrm{CD}_{X}\right)$.

Remark 5. For a topological space $X$ and $x \in X$, Gruenhage's $W$-game for $X$ at $x$ is $G_{1}\left(\mathscr{N}(x), \neg \Gamma_{X, x}\right)$ and Gruenhage's clustering game for $X$ at $x$ is $G_{1}\left(\mathscr{N}(x), \neg \Omega_{X, x}\right)$.

Definition 14. Suppose $(P, \leqslant)$ is a partially ordered set and $\mathcal{A}, \mathcal{B} \subseteq P$. Then $\mathcal{A}$ has cofinality $\kappa$ relative to $\mathcal{B}$, denoted

$$
\operatorname{cof}(\mathcal{A} ; \mathcal{B}, \leqslant)=\kappa,
$$

if $\kappa$ is the minimum cardinal so that there is a collection $\left\{A_{\alpha}: \alpha<\kappa\right\} \subseteq \mathcal{A}$ with the property that whenever $B \in \mathcal{B}$, there is an $\alpha$ so that $B \leqslant A_{\alpha}$. If there is no such cardinal don't define the cofinality.

Definition 15. Suppose $(P, \leqslant)$ and $\left(Q, \leqslant^{*}\right)$ are partial orders and $\mathcal{A}, \mathcal{B} \subseteq P, \mathcal{C}, \mathcal{D} \subseteq Q$. Then

$$
(\mathcal{A} ; \mathcal{B}, \leqslant) \geqslant_{T}\left(\mathcal{C} ; \mathcal{D}, \leqslant^{*}\right)
$$

if there is a map $\varphi: \mathcal{A} \rightarrow \mathcal{C}$ so that whenever $\mathcal{F} \subseteq \mathcal{A}$ is cofinal relative to $\mathcal{B}$, then $\varphi[\mathcal{F}]$ is cofinal relative to $\mathcal{D}$. This definition is inspired by Paul Gartside and Ana Mamatelashvili's work on the Tukey order [5].

Suppose $(P, \leqslant)$ is a partially ordered set. We define $\leqslant$ on $P \times \omega$ by

$$
(p, n) \leqslant(q, m) \Longleftrightarrow(p \leqslant q \text { and } n \leqslant m) .
$$

Lemma 1. For any partially ordered set $(P, \leqslant)$ and any $Q \subseteq P,(Q \times \omega, P \times \omega) \geqslant_{T}(Q, P)$. 
Proof. Let $\phi: P \times \omega \rightarrow P$ be defined by $\phi(p, n)=p$. Suppose $A \subseteq P \times \omega$ is cofinal for $Q \times \omega$ and let $q \in Q$ be arbitrary. By the cofinality of $A$, we can find $(r, m) \in A$ so that $(q, 0) \leqslant(r, m)$. It follows that $q \leqslant r=\phi(r, m)$ which demonstrates that $\phi[A]$ is cofinal for $Q$.

Lemma 2. Suppose $(P, \leqslant)$ and $\left(Q, \leqslant^{*}\right)$ are partial orders, $\mathcal{A}, \mathcal{B} \subseteq P$, and $\mathcal{C}, \mathcal{D} \subseteq Q$. Suppose further that $(\mathcal{A} ; \mathcal{B}, \leqslant)=_{T}\left(\mathcal{C} ; \mathcal{D}, \leqslant^{*}\right)$ and $\operatorname{cof}(\mathcal{A} ; \mathcal{B}, \leqslant)=\kappa$. Then $\operatorname{cof}\left(\mathcal{C} ; \mathcal{D}, \leqslant^{*}\right)=\kappa$.

Proof. Let $\varphi: \mathcal{A} \rightarrow \mathcal{C}$ be so that whenever $\mathcal{F} \subseteq \mathcal{A}$ is cofinal for $\mathcal{B}$, then $\varphi[\mathcal{F}]$ is cofinal for $\mathcal{D}$. Also let $\mathcal{F}=\left\{A_{\alpha}: \alpha<\kappa\right\} \subseteq \mathcal{A}$ be cofinal for $\mathcal{B}$. Then $\varphi[\mathcal{F}]$ is a subset of $\mathcal{C}$ and is cofinal for $\mathcal{D}$. Thus $\operatorname{cof}\left(\mathcal{C} ; \mathcal{D}, \leqslant^{*}\right) \leqslant \kappa$.

Suppose towards a contradiction that $\operatorname{cof}\left(\mathcal{C} ; \mathcal{D}, \leqslant^{*}\right)=\lambda<\kappa$. Then we can find a collection $\mathcal{G}=\left\{C_{\alpha}: \alpha<\lambda\right\} \subseteq \mathcal{C}$ which is cofinal for $\mathcal{D}$. Now let $\psi: \mathcal{C} \rightarrow \mathcal{A}$ witness that $\left(\mathcal{C} ; \mathcal{D}, \leqslant^{*}\right) \geqslant_{T}$ $(\mathcal{A} ; \mathcal{B}, \leqslant)$. Then $\psi[\mathcal{G}] \subseteq \mathcal{A}$ and is cofinal for $\mathcal{B}$. But this would imply that $\operatorname{cof}(\mathcal{A} ; \mathcal{B}, \leqslant)<\kappa$, a contradiction.

Lemma 3. Suppose $X$ is a Tychonoff space. Assume $\mathcal{A}, \mathcal{B} \subseteq \wp(X)$. Then

$$
\left(\mathscr{N}_{C_{\mathcal{A}}(X)}(\mathbf{0}) ; \mathscr{N}_{C_{\mathcal{B}}(X)}(\mathbf{0}), \supseteq\right) \leqslant T(\mathcal{A} \times \omega ; \mathcal{B} \times \omega, \subseteq)
$$

and

$$
\left(\mathscr{N}_{C_{\overline{\mathcal{A}}}(X)}(\mathbf{0}) ; \mathscr{N}_{C_{\mathcal{B}}(X)}(\mathbf{0}), \supseteq\right)=_{T}(\overline{\mathcal{A}} \times \omega ; \mathcal{B} \times \omega, \subseteq) .
$$

Proof. To address $\left(\mathscr{N}_{C_{\mathcal{A}}(X)}(\mathbf{0}) ; \mathscr{N}_{C_{\mathcal{B}}(X)}(\mathbf{0}), \supseteq\right) \leqslant_{T}(\mathcal{A} \times \omega ; \mathcal{B} \times \omega, \subseteq)$, define $\psi: \mathcal{A} \times \omega \rightarrow \mathscr{N}_{C_{\mathcal{A}}}(\mathbf{0})$ by

$$
\psi(A, n)=\left[\mathbf{0} ; A, 2^{-n}\right] .
$$

Suppose $\mathcal{F} \subseteq \mathcal{A} \times \omega$ is cofinal for $\mathcal{B} \times \omega$ and let $U \in \mathscr{N}_{C_{\mathcal{B}}(X)}(\mathbf{0})$ be arbitrary. We can find $B \in \mathcal{B}$ and $n \in \omega$ so that

$$
\left[\mathbf{0} ; B, 2^{-n}\right] \subseteq U .
$$

By the cofinality of $\mathcal{F}$ relative to $\mathcal{B} \times \omega$, we can find $A \in \mathcal{A}$ and $m \in \omega$ so that $B \subseteq A$ and $n \leqslant m$. It follows that

$$
\psi(A, m)=\left[\mathbf{0} ; A, 2^{-m}\right] \subseteq\left[\mathbf{0} ; B, 2^{-n}\right] \subseteq U .
$$

That is, $\psi[\mathcal{F}]$ is cofinal in $\mathscr{N}_{C_{\mathcal{B}}(X)}(\mathbf{0})$.

Without loss of generality, suppose $\mathcal{A}=\overline{\mathcal{A}}$. To address

$$
\left(\mathscr{N}_{C_{\mathcal{A}}(X)}(\mathbf{0}) ; \mathscr{N}_{C_{\mathcal{B}}(X)}(\mathbf{0}), \supseteq\right) \geqslant_{T}(\mathcal{A} \times \omega ; \mathcal{B} \times \omega, \subseteq),
$$

let $\phi: \mathscr{N}_{C_{\mathcal{A}}(X)}(\mathbf{0}) \rightarrow \mathcal{A} \times \omega$ be defined in the following way. For any $U \in \mathscr{N}_{C_{\mathcal{A}}(X)}(\mathbf{0})$, let $A_{U} \in \mathcal{A}$ and $\varepsilon_{U}>0$ be so that

$$
\left[\mathbf{0} ; A_{U}, \varepsilon_{U}\right] \subseteq U
$$

Choose $n_{U} \in \omega$ so that $2^{-n_{U}}<\varepsilon_{U}$. Then define $\phi(U)=\left\langle A_{U}, n_{U}\right\rangle$.

Suppose $\mathcal{F} \subseteq \mathscr{N}_{C_{\mathcal{A}}(X)}(\mathbf{0})$ is cofinal for $\mathscr{N}_{C_{\mathcal{B}}(X)}(\mathbf{0})$. To see that $\phi[\mathcal{F}]$ is cofinal for $\mathcal{B} \times \omega$, let $B \in \mathcal{B}$ and $n \in \omega$. Then $\left[\mathbf{0} ; B, 2^{-n}\right] \in \mathscr{N}_{C_{\mathcal{B}}(X)}(\mathbf{0})$ which means there exists some $U \in \mathcal{F}$ so that $U \subseteq\left[\mathbf{0} ; B, 2^{-n}\right]$. Moreover,

$$
\left[\mathbf{0} ; A_{U}, 2^{-n_{U}}\right] \subseteq U \subseteq\left[\mathbf{0} ; B, 2^{-n}\right] .
$$

Suppose toward contradiction that $B \nsubseteq A_{U}$. Then, for $x \in B \backslash A_{U}$, we can find a continuous function $f: X \rightarrow[0,1]$ so that $f(x)=1$ and $f \uparrow_{A_{U}} \equiv 0$. But then $f \in\left[\mathbf{0} ; A_{U}, 2^{-n_{U}}\right] \backslash\left[\mathbf{0} ; B, 2^{-n}\right]$, a contradiction.

Were $n>n_{U}$, consider the constant function defined by $f(x)=2^{-n}$. This is a contradiction to $\left[\mathbf{0} ; A_{U}, 2^{-n_{U}}\right] \subseteq\left[\mathbf{0} ; B, 2^{-n}\right]$ so $n \leqslant n_{U}$.

Since $B \subseteq A_{U}$ and $n \leqslant n_{U}$, we see that $\phi[\mathcal{F}]$ is cofinal for $\mathcal{B} \times \omega$. 


\section{Strengthening Strategies}

Lemma 4. Suppose $\mathcal{A}$ is an ideal-base, $X=\bigcup \mathcal{A}$, and let $\mathscr{U} \in \mathcal{O}(X, \mathcal{A})$. Then, for each $A \in \mathcal{A}$, $\{U \in \mathscr{U}: A \in U\}$ is infinite. That is, $\mathcal{O}(X, \mathcal{A})=\Lambda(X, \mathcal{A})$.

Proof. Let $A \in \mathcal{A}$ be arbitrary and let $U_{0} \in \mathscr{U}$ be so that $A \subseteq U_{0}$. Since $X \backslash U_{0} \neq \emptyset$, let $x_{1} \in X \backslash U_{0}$ and let $A_{1}^{*} \in \mathcal{A}$ be so that $x_{1} \in A_{1}^{*}$. Let $A_{1} \in \mathcal{A}$ be so that $A \cup A_{1}^{*} \subseteq A_{1}$ and let $U_{1} \in \mathscr{U}$ be so that $A_{1} \subseteq U_{1}$. Since $A_{1} \cap\left(X \backslash U_{0}\right) \neq \emptyset$, we know that $U_{0} \neq U_{1}$. Inductively continue in this way.

Corollary 5. Suppose $\mathcal{A}$ and $\mathcal{B}$ are ideal-bases. Then $G_{1}(\mathscr{N}[\mathcal{A}], \neg \mathcal{O}(X, \mathcal{B}))$ is equivalent to $G_{1}(\mathscr{N}[\mathcal{A}], \neg \Lambda(X, \mathcal{B}))$.

Definition 16. For collections $\mathcal{A}$ and $\mathcal{B}$, recall that $\mathcal{A}$ refines $\mathcal{B}$, denoted $\mathcal{A} \prec \mathcal{B}$, provided that, for every $B \in \mathcal{B}$, there exists $A \in \mathcal{A}$ so that $A \subseteq B$.

Lemma 6. $\mathcal{A} \prec \mathcal{B}$ if and only if $\mathcal{O}(X, \mathcal{B}) \subseteq \mathcal{O}(X, \mathcal{A})$.

Proof. Suppose $\mathcal{A} \prec \mathcal{B}$. Let $\mathscr{U} \in \mathcal{O}(X, \mathcal{B})$ and $A \in \mathcal{A}$. Let $B \in \mathcal{B}$ be so that $A \subseteq B$ and let $U \in \mathscr{U}$ be so that $B \subseteq U$. You get the idea.

Now, suppose $\mathcal{A} \nprec \mathcal{B}$. Let $A \in \mathcal{A}$ be so that, for all $B \in \mathcal{B}, A \nsubseteq B$. Then choose $x_{B} \in A \backslash B$ and set $U_{B}=X \backslash\left\{x_{B}\right\}$ for each $B \in \mathcal{B}$. Notice that $B \subseteq U_{B}$ so $\left\{U_{B}: B \in \mathcal{B}\right\} \in \mathcal{O}(X, \mathcal{B})$. Clearly, $\left\{U_{B}: B \in \mathcal{B}\right\} \notin \mathcal{O}(X, \mathcal{A})$.

In [9], Pawlikowski showed that $S_{\text {fin }}\left(\mathcal{O}_{X}, \mathcal{O}_{X}\right)$ if and only if I $\forall G_{\text {fin }}\left(\mathcal{O}_{X}, \Lambda_{X}\right)$ and also that $S_{1}\left(\mathcal{O}_{X}, \mathcal{O}_{X}\right)$ if and only if I $\forall G_{1}\left(\mathcal{O}_{X}, \Lambda_{X}\right)$. The authors generalized this in a previous paper. The following lemmas are slightly more general than proved there, but the proofs are the same as in [1].

Lemmas 7 and 8 are only known to be true if both cover types are $\omega$-covers or if both cover types are $k$-covers. See arXiv:2102.00296 for a proof of the $k$-covers case.

Lemma 7. Assume $\mathcal{A} \prec \mathcal{B}$ and $S_{\text {fin }}(\mathcal{O}(X, \mathcal{A}), \mathcal{O}(X, \mathcal{B}))$. Then I $\bigvee G_{\text {fin }}(\mathcal{O}(X, \mathcal{A}), \Lambda(X, \mathcal{B}))$. Moreover, I $\uparrow G_{\text {fin }}(\mathcal{O}(X, \mathcal{A}), \mathcal{O}(X, \mathcal{B}))$ if and only if I $\uparrow G_{\text {pre }}(\mathcal{O}(X, \mathcal{A}), \mathcal{O}(X, \mathcal{B}))$.

Lemma 8. Assume $\mathcal{A} \prec \mathcal{B}$ and $S_{1}(\mathcal{O}(X, \mathcal{A}), \mathcal{O}(X, \mathcal{B}))$. Then I $\bigvee G_{1}(\mathcal{O}(X, \mathcal{A}), \Lambda(X, \mathcal{B}))$. Moreover, $\mathrm{I} \uparrow G_{1}(\mathcal{O}(X, \mathcal{A}), \mathcal{O}(X, \mathcal{B}))$ if and only if $\mathrm{I}_{\text {pre }}^{\uparrow} G_{1}(\mathcal{O}(X, \mathcal{A}), \mathcal{O}(X, \mathcal{B}))$.

In [12], Tkachuk showed that $\mathrm{I} \uparrow G_{1}\left([X]^{<\omega}, \neg \mathcal{O}_{X}\right)$ if and only if $\mathrm{I} \uparrow G_{1}\left([X]^{<\omega}, \neg \Gamma_{X}\right)$. The authors generalized this result to $\mathcal{O}(X, \mathcal{A})$ in [1], assuming that $\mathcal{A}$ is an ideal. Here we show that one only needs to assume that $\mathcal{A}$ is an ideal base.

Lemma 9. For any strategy $\sigma$ for One in $G_{1}(\mathcal{A}, \mathcal{B})$ where $\mathcal{A}$ and $\mathcal{B}$ are collections, define

$$
\operatorname{play}_{\sigma}=\left\{\left\langle x_{0}, x_{1}, \ldots, x_{n}\right\rangle:(n \in \omega) \wedge(\forall \ell<n)\left[x_{\ell} \in \sigma\left(\left\langle x_{j}: j<\ell\right\rangle\right)\right]\right\} \subseteq(\bigcup \mathcal{A})^{<\omega}
$$

and

$$
\operatorname{play}_{\sigma}^{\omega}=\left\{\left\langle x_{n}: n \in \omega\right\rangle:(\forall n \in \omega)\left[\left\langle x_{\ell}: \ell \leqslant n\right\rangle \in \operatorname{play}_{\sigma}\right]\right\} \subseteq(\bigcup \mathcal{A})^{\omega}
$$

If $\sigma$ is a winning strategy, then for any $\left\langle x_{n}: n \in \omega\right\rangle \in$ play $_{\sigma}^{\omega},\left\{x_{n}: n \in \omega\right\} \notin \mathcal{B}$.

Proof. Let $\left\langle x_{n}: n \in \omega\right\rangle \in$ play $_{\sigma}^{\omega}$. Let $A_{0}=\sigma(\emptyset)$ and notice that $x_{0} \in A_{0}$ since $\left\langle x_{0}\right\rangle \in$ play $_{\sigma}$. Now suppose we have $A_{0}, A_{1}, \ldots, A_{n} \in \mathcal{A}$ defined so that $x_{\ell} \in A_{\ell}=\sigma\left(\left\langle x_{j}: j<\ell\right\rangle\right)$. Let $A_{n+1}=$ $\sigma\left(\left\langle x_{0}, x_{1}, \ldots, x_{n}\right\rangle\right)$. We claim that $x_{n+1} \in A_{n+1}$. To see this, we know that $\left\langle x_{0}, x_{1}, \ldots, x_{n+1}\right\rangle \in$ play $_{\sigma}$ so $x_{n+1} \in \sigma\left(\left\langle x_{j}: j<n+1\right\rangle\right)=A_{n+1}$. Hence, the $x_{n}$ arise from a single run of the game according to $\sigma$.

Since $\sigma$ is winning for One, $\left\{x_{n}: n \in \omega\right\} \notin \mathcal{B}$. 
Proposition 10. Let $\mathcal{A}$ and $\mathcal{B}$ be collections. Set

$$
\mathcal{B}_{\Gamma}=\left\{B \in \mathcal{B}:\left(\text { for all infinite } B^{\prime} \subseteq B\right)\left[B^{\prime} \in \mathcal{B}\right]\right\}
$$

If $\mathcal{A}$ is a filter base, then $\mathrm{I} \uparrow G_{1}(\mathcal{A}, \neg \mathcal{B})$ if and only if $\mathrm{I} \uparrow G_{1}\left(\mathcal{A}, \neg \mathcal{B}_{\Gamma}\right)$.

Proof. Let $s$ be a winning strategy for One in $G_{1}(\mathcal{A}, \neg \mathcal{B})$. For $\left\langle x_{0}, \cdots, x_{n}\right\rangle \in$ play $_{s}$, define $\gamma\left(x_{0}, \cdots, x_{n}\right) \in \mathcal{A}$ to be so that

$$
\gamma\left(x_{0}, \cdots, x_{n}\right) \subseteq \bigcap_{j=0}^{n} s\left(x_{0}, \cdots, x_{j}\right) .
$$

Now we will define a winning strategy $\sigma$ for One in $G_{1}\left(\mathcal{A}, \neg \mathcal{B}_{\Gamma}\right)$. First set $\sigma(\emptyset)=s(\emptyset)=A_{0}$. Now suppose we have defined $\sigma\left(x_{0}, \cdots, x_{n-1}\right)$ for all $x_{0}, \cdots, x_{n-1}$ satisfying $x_{0} \in \sigma(\emptyset), x_{1} \in \sigma\left(x_{0}\right)$, and so on. Suppose also that $\sigma$ has been defined in such a way that for a fixed $x_{n} \in \sigma\left(x_{0}, \cdots, x_{n-1}\right)$,

(i) for any $0 \leqslant j_{0}<j_{1}<\cdots<j_{k} \leqslant n,\left\langle x_{j_{0}}, x_{j_{1}}, \cdots, x_{j_{k}}\right\rangle \in$ play $_{s}$, and

(ii) for any $0 \leqslant j_{0}<j_{1}<\cdots<j_{k} \leqslant \ell<n, x_{\ell+1} \in \gamma\left(x_{j_{0}}, x_{j_{1}}, \cdots, x_{j_{k}}\right)$.

Define $A_{n+1} \in \mathcal{A}$ to be so that

$$
A_{n+1} \subseteq \bigcap\left\{\gamma\left(x_{j_{0}}, x_{j_{1}}, \cdots, x_{j_{k}}\right): 0 \leqslant j_{0}<j_{1}<\cdots<j_{k} \leqslant n\right\}
$$

Then set $\sigma\left(x_{0}, \cdots, x_{n}\right)=A_{n+1}$.

We check that this definition satisfies the two properties relative to $n+1$. Fix $x_{n+1} \in A_{n+1}$. Let $0 \leqslant j_{0}<j_{1}<\cdots<j_{k} \leqslant n+1$. Notice that $\left\langle x_{j_{0}}, x_{j_{1}}, \cdots, x_{j_{k-1}}\right\rangle \in$ play $_{s}$ by the inductive hypothesis. So let $A_{j_{m}}^{*}=s\left(x_{j_{0}}, \cdots, x_{j_{m}}\right)$ for $0 \leqslant m<k$ and

$$
A_{j_{k}}^{*}=s\left(x_{j_{0}}, x_{j_{1}}, \cdots, x_{j_{k-1}}\right) \text {. }
$$

It follows that $A_{n+1} \subseteq A_{j_{k}}^{*}$ and that $x_{n+1} \in A_{j_{k}}^{*}$. Hence,

$$
A_{j_{0}}^{*}, x_{j_{0}}, \cdots, A_{j_{k}}^{*}, x_{j_{k}}
$$

is a play according to $s$.

The second property holds by the definition of $\sigma$. This completes the definition of $\sigma$.

We now show that $\sigma$ is a winning strategy. Suppose $A_{0}, x_{0}, A_{1}, x_{1}, \cdots$ is a full run of the game $G_{1}\left(\mathcal{A}, \neg \mathcal{B}_{\Gamma}\right)$ played according to $\sigma$. Suppose, by way of contradiction, that there is an infinite $B^{\prime} \subseteq\left\{x_{n}: n \in \omega\right\}$ so that $B^{\prime} \notin \mathcal{B}$. Say $B^{\prime}=\left\{x_{j_{n}}: n \in \omega\right\}$. Then by the construction of $\sigma$, $\left\langle x_{0}, \cdots, x_{j_{n}}\right\rangle \in$ play $_{s}$ for all $n \in \omega$. Hence, $\left\{x_{j_{n}}: n \in \omega\right\} \in$ play $_{s}^{\omega}$, and so by the Lemma 9 , $\left\{x_{j_{n}}: n \in \omega\right\}=B^{\prime} \in \mathcal{B}$, a contradiction. Thus $\left\{x_{n}: \in \omega\right\} \in \mathcal{B}_{\Gamma}$, and $\sigma$ is a winning strategy.

The other direction of the proof is obvious.

Corollary 11. Let $\mathcal{A}$ be an ideal-base. Then One has winning (pre-determined) strategy for the game $G_{1}(\mathscr{N}[\mathcal{A}], \neg \mathcal{O}(X, \mathcal{B}))$ if and only if One has winning (pre-determined) strategy for $G_{1}(\mathscr{N}[\mathcal{A}], \neg \Gamma(X, \mathcal{B}))$. The same is true for pre-determined strategies.

Proof. Notice that if $\mathcal{A}$ is an ideal base, then $\mathscr{N}[\mathcal{A}]$ is a filter base. Also notice that $\mathcal{O}(X, \mathcal{B})_{\Gamma}$ is the same thing as $\Gamma(X, \mathcal{B})$. This shows that $\mathrm{I} \uparrow G_{1}(\mathscr{N}[\mathcal{A}], \neg \mathcal{O}(X, \mathcal{B})) \Longleftrightarrow \mathrm{I} \uparrow G_{1}(\mathscr{N}[\mathcal{A}], \neg \Gamma(X, \mathcal{B}))$.

The fact that the results hold for pre-determined strategies follows from a modification of the proof of the proposition. Simply set

$$
\sigma(n)=s(0) \cap \cdots \cap s(n)
$$

and check that this works. 


\section{An Order on Single Selection Games}

Definition 17. Let $\mathcal{A}, \mathcal{B}, \mathcal{C}$, and $\mathcal{D}$ be collections and $\alpha$ be an ordinal. Say that $G_{1}^{\alpha}(\mathcal{A}, \mathcal{C}) \leqslant \mathrm{II}$ $G_{1}^{\alpha}(\mathcal{B}, \mathcal{D})$ if

- II $\underset{\text { mark }}{\uparrow} G_{1}^{\alpha}(\mathcal{A}, \mathcal{C}) \Longrightarrow \mathrm{II} \underset{\text { mark }}{\uparrow} G_{1}^{\alpha}(\mathcal{B}, \mathcal{D})$,

- II $\uparrow G_{1}^{\alpha}(\mathcal{A}, \mathcal{C}) \Longrightarrow \operatorname{II} \uparrow G_{1}^{\alpha}(\mathcal{B}, \mathcal{D})$,

- I $\bigvee G_{1}^{\alpha}(\mathcal{A}, \mathcal{C}) \Longrightarrow \operatorname{I} \ngtr G_{1}^{\alpha}(\mathcal{B}, \mathcal{D})$, and

- I $\bigwedge_{\text {pre }} G_{1}^{\alpha}(\mathcal{A}, \mathcal{C}) \Longrightarrow \mathrm{I} \underset{\text { pre }}{\uparrow} G_{1}^{\alpha}(\mathcal{B}, \mathcal{D})$.

Notice that if $G_{1}^{\alpha}(\mathcal{A}, \mathcal{C}) \leqslant{ }_{I I} G_{1}^{\alpha}(\mathcal{B}, \mathcal{D})$ and $G_{1}^{\alpha}(\mathcal{B}, \mathcal{D}) \leqslant{ }_{I I} G_{1}^{\alpha}(\mathcal{A}, \mathcal{C})$, then the games are equivalent. Also notice that $\leqslant$ II is transitive.

Theorem 12. Let $\mathcal{A}, \mathcal{B}, \mathcal{C}$, and $\mathcal{D}$ be collections and $\alpha$ be an ordinal. Suppose there are functions

- $\overleftarrow{T}_{\mathrm{I}, \xi}: \mathcal{B} \rightarrow \mathcal{A}$ and

- $\vec{T}_{\mathrm{II}, \xi}: \bigcup \mathcal{A} \times \mathcal{B} \rightarrow \cup \mathcal{B}$

for each $\xi \in \alpha$, so that

(Tr1) If $x \in \overleftarrow{T}_{\mathrm{I}, \xi}(B)$, then $\vec{T}_{\mathrm{II}, \xi}(x, B) \in B$

$(\operatorname{Tr} 2)$ If $x_{\xi} \in \overleftarrow{T}_{\mathrm{I}, \xi}\left(B_{\xi}\right)$ and $\left\{x_{\xi}: \xi \in \alpha\right\} \in \mathcal{C}$, then $\left\{\vec{T}_{\mathrm{II}, \xi}\left(x_{\xi}, B_{\xi}\right): \xi \in \alpha\right\} \in \mathcal{D}$

Then $G_{1}^{\alpha}(\mathcal{A}, \mathcal{C}) \leqslant{ }_{\text {II }} G_{1}^{\alpha}(\mathcal{B}, \mathcal{D})$.

Proof. Suppose II $\underset{\text { mark }}{\uparrow} G_{1}^{\alpha}(\mathcal{A}, \mathcal{C})$ and let $\tau$ be a winning Markov strategy for Two. We define a winning Markov strategy for Two in $G_{1}^{\alpha}(\mathcal{B}, \mathcal{D})$. Toward this end, let $\left\{B_{\xi}: \xi \in \alpha\right\} \subseteq \mathcal{B}$ be arbitrary and set $A_{\xi}=\overleftarrow{T}_{\mathrm{I}, \xi}\left(B_{\xi}\right)$ and $x_{\xi}=\tau\left(A_{\xi}, \xi\right)$. Define $y_{\xi}=\vec{T}_{\mathrm{II}, \xi}\left(x_{\xi}, B_{\xi}\right)$. Then

$$
\left\{x_{\xi}: \xi \in \alpha\right\} \in \mathcal{C} \Longrightarrow\left\{y_{\xi}: \xi \in \alpha\right\} \in \mathcal{D} \text {. }
$$

Suppose II $\uparrow G_{1}^{\alpha}(\mathcal{A}, \mathcal{C})$ and let $\tau$ be a winning strategy for Two. We define a strategy $t$ for Two in $G_{1}^{\alpha}(\mathcal{B}, \mathcal{D})$ recursively. Suppose One plays $B_{0}$. Then $A_{0}:=\overleftarrow{T}_{\mathrm{I}, 0}\left(B_{0}\right)$ is an initial play of $G_{1}^{\alpha}(\mathcal{A}, \mathcal{C})$. So $x_{0}:=\tau\left(A_{0}\right) \in A_{0}$. Define

$$
t\left(B_{0}\right)=y_{0}=\vec{T}_{\mathrm{II}, 0}\left(x_{0}, B_{0}\right) .
$$

For $\beta \in \alpha$, suppose we have $\left\{A_{\xi}: \xi<\beta\right\},\left\{B_{\xi}: \xi<\beta\right\},\left\{x_{\xi}: \xi<\beta\right\}$, and $\left\{y_{\xi}: \xi<\beta\right\}$ defined. Given $B_{\beta} \in \mathcal{B}$, let $A_{\beta}=\overleftarrow{T}_{\mathrm{I}, \beta}\left(B_{\beta}\right)$ and $x_{\beta}=\tau\left(A_{0}, \ldots, A_{\beta}\right) \in A_{\beta}$. Then set

$$
t\left(B_{0}, \ldots, B_{\beta}\right)=y_{\beta}=\vec{T}_{\mathrm{II}, \beta}\left(x_{\beta}, B_{\beta}\right) .
$$

This concludes the definition of $t$. By $(\operatorname{Tr} 1)$, since $x_{\xi} \in \overleftarrow{T}_{\mathrm{I}, \xi}\left(B_{\xi}\right)$, it follows that $y_{\xi} \in B_{\xi}$. Using $(\operatorname{Tr} 2)$, we see that

$$
\left\{x_{\xi}: \xi \in \alpha\right\} \in \mathcal{C} \Longrightarrow\left\{y_{\xi}: \xi \in \alpha\right\} \in \mathcal{D} \text {. }
$$


Suppose I $\uparrow G_{1}^{\alpha}(\mathcal{B}, \mathcal{D})$ and let $\sigma$ witness this. We will develop a strategy $s$ for One in $G_{1}^{\alpha}(\mathcal{A}, \mathcal{B})$. Let $B_{0}=\sigma(\emptyset)$ and $s(\emptyset)=A_{0}=\overleftarrow{T}_{\mathrm{I}, 0}\left(B_{0}\right)$. Then, for $\beta \in \alpha$, suppose we have $\left\{A_{\xi}: \xi \leqslant \beta\right\} \subseteq \mathcal{A}$, $\left\{B_{\xi}: \xi \leqslant \beta\right\} \subseteq \mathcal{B},\left\{x_{\xi}: \xi<\beta\right\}$, and $\left\{y_{\xi}: \xi<\beta\right\}$ defined in the right way. Suppose $x_{\beta} \in A_{\beta}$. Then set $y_{\beta}=\vec{T}_{\mathrm{II}, \beta}\left(x_{\beta}, B_{\beta}\right) \in B_{\beta}, B_{\beta+1}=\sigma\left(y_{0}, \ldots, y_{\beta}\right)$ and

$$
s\left(x_{0}, \ldots, x_{\beta}\right)=A_{\beta+1}=\overleftarrow{T}_{\mathrm{I}, \beta+1}\left(B_{\beta+1}\right)
$$

After the run of the game is completed, let $x_{\xi+1} \in s\left(x_{0}, \cdots, x_{\xi}\right)$ for all $\xi \in \alpha$ and $x_{0} \in s(\emptyset)$. Then (Tr1) gives us that $\vec{T}_{\mathrm{II}, \xi}\left(x_{\xi}, B_{\xi}\right)=y_{\xi} \in B_{\xi}$. As $\sigma$ is a winning strategy for One in $\mathrm{I} \uparrow G_{1}^{\alpha}(\mathcal{B}, \mathcal{D})$, (Tr2) yields

$$
\left\{y_{\xi}: \xi \in \alpha\right\} \notin \mathcal{D} \Longrightarrow\left\{x_{\xi}: \xi \in \alpha\right\} \notin \mathcal{C}
$$

Suppose I $\underset{\text { pre }}{\uparrow} G_{1}^{\alpha}(\mathcal{B}, \mathcal{D})$ and let $\left\{B_{\xi}: \xi \in \alpha\right\}$ represent One's winning strategy. Let $A_{\xi}=$ $\overleftarrow{T}_{\mathrm{I}, \xi}\left(B_{\xi}\right)$ for each $\xi \in \alpha$. We will show that $\left\{A_{\xi}: \xi \in \alpha\right\}$ forms a winning strategy for One in $G_{1}^{\alpha}(\mathcal{A}, \mathcal{C})$. Let $x_{\xi} \in A_{\xi}$ for all $\xi \in \alpha$ and let $y_{\xi}=\vec{T}_{\mathrm{II}, \xi}\left(x_{\xi}, B_{\xi}\right)$. By $(\operatorname{Tr} 1), y_{\xi} \in B_{\xi}$ for all $\xi \in \alpha$ and so $\left\{y_{\xi}: \xi \in \alpha\right\} \notin \mathcal{D}$. By $(\operatorname{Tr} 2)$, we see that $\left\{x_{\xi}: \xi \in \alpha\right\} \notin \mathcal{C}$.

In some situations, the use of both maps is not necessary as the translation between player One's moves simply comes from lifting the translation of player Two's selections.

Corollary 13. Let $\mathcal{A}, \mathcal{B}, \mathcal{C}$, and $\mathcal{D}$ be collections. Suppose there is a map $\phi:(\bigcup \mathcal{B}) \times \omega \rightarrow(\bigcup \mathcal{A})$ so that

- For all $B \in \mathcal{B}$ and all $n \in \omega,\{\phi(y, n): y \in B\} \in \mathcal{A}$

- if $\left\{\phi\left(y_{n}, n\right): n \in \omega\right\} \in \mathcal{C}$, then $\left\{y_{n}: n \in \omega\right\} \in \mathcal{D}$

Then $G_{1}(\mathcal{A}, \mathcal{C}) \leqslant \mathrm{II} G_{1}(\mathcal{B}, \mathcal{D})$.

Proof. Define $\overleftarrow{T}_{\mathrm{I}, n}: \mathcal{B} \rightarrow \mathcal{A}$ by

$$
\overleftarrow{T}_{\mathrm{I}, n}(B)=\phi[B \times\{n\}]
$$

From the first assumption on $\phi$ we know that $\overleftarrow{T}_{\mathrm{I}, n}$ really does produce objects in $\mathcal{A}$. For $x \in$ $\phi[B \times\{n\}]$ and $n \in \omega$, choose $y_{x, n} \in B$ so that $\phi\left(y_{x, n}, n\right)=x$. Define $\vec{T}_{\text {II }, n}: \bigcup \mathcal{A} \times \mathcal{B} \rightarrow \bigcup \mathcal{B}$ by

$$
\vec{T}_{\mathrm{II}, n}(x)(B)=y_{x, n}
$$

if possible and otherwise set it to be an arbitrary element of $\bigcup \mathcal{B}$. So if $x \in \overleftarrow{T}_{\mathrm{I}, n}(B)$, then $\vec{T}_{\text {II }, n}(x)(B)=y_{x, n} \in B$.

Now suppose $x_{n} \in \phi\left[B_{n} \times\{n\}\right]$ and $\left\{x_{n}: n \in \omega\right\} \in \mathcal{C}$. Then $\left\{\phi\left(y_{x_{n}, n}, n\right): n \in \omega\right\} \in \mathcal{C}$. By the second assumption on $\phi$, it follows that $\left\{y_{x_{n}}: n \in \omega\right\} \in \mathcal{D}$. Thus $\left\{\vec{T}_{\text {II }, n}\left(x_{n}\right)\left(B_{n}\right): n \in \omega\right\} \in \mathcal{D}$. This completes the proof.

\section{Equivalent and Dual Classes of Games}

Corollary 14. Let $X$ be a Tychonoff space and $\mathcal{A}, \mathcal{B} \subseteq \wp(X)$. Then

(i) $G_{1}(\mathcal{O}(X, \mathcal{A}), \Lambda(X, \mathcal{B})) \leqslant{ }_{I I} G_{1}\left(\Omega_{C_{\mathcal{A}}(X), \mathbf{0}}, \Omega_{C_{\mathcal{B}}(X), \mathbf{0}}\right)$,

(ii) $G_{1}\left(\Omega_{C_{\mathcal{A}}(X), \mathbf{0}}, \Omega_{C_{\mathcal{B}}(X), \mathbf{0}}\right) \leqslant{ }_{\mathrm{II}} G_{1}\left(\mathcal{D}_{C_{\mathcal{A}}(X)}, \Omega_{C_{\mathcal{B}}(X), \mathbf{0}}\right)$, and 
(iii) if $\mathcal{A}$ consists of closed sets and $X$ is $\mathcal{A}$-normal, then

$$
G_{1}\left(\mathcal{D}_{C_{\mathcal{A}}(X)}, \Omega_{C_{\mathcal{B}}(X), 0}\right) \leqslant{ }_{\mathrm{II}} G_{1}(\mathcal{O}(X, \mathcal{A}), \Lambda(X, \mathcal{B}))
$$

Thus if $\mathcal{A}$ consists of closed sets and $X$ is $\mathcal{A}$-normal, then the three games are equivalent.

Proof. Let $\phi: C_{\mathcal{A}}(X) \times \omega \rightarrow \mathscr{T}_{X}$ be defined by $\phi(f, n)=f^{-1}\left[\left(-2^{-n}, 2^{-n}\right)\right]$. Suppose $F \in \Omega_{C_{\mathcal{A}}(X), \mathbf{0}}$ and let both $A \in \mathcal{A}$ and $n \in \omega$ be arbitrary. Choose $f \in F$ so that $f \in\left[\mathbf{0} ; A, 2^{-n}\right]$ and notice that $A \subseteq f^{-1}\left[\left(-2^{-n}, 2^{-n}\right)\right]$. Hence, $\{\phi(f, n): f \in F\} \in \mathcal{O}(X, \mathcal{A})$.

Next, suppose $\left\{\phi\left(f_{n}, n\right): n \in \omega\right\} \in \Lambda(X, \mathcal{B})$. Let $B \in \mathcal{B}$ and $\varepsilon>0$ be arbitrary. Then, there is $n \in \omega$ large enough so that $B \subseteq f_{n}^{-1}\left[\left(-2^{-n}, 2^{-n}\right)\right]$ and $2^{-n}<\varepsilon$. It follows that $f \in[\mathbf{0} ; B, \varepsilon]$. By Corollary 13, this completes (i).

Next we check that $G_{1}\left(\Omega_{C_{\mathcal{A}}(X), \mathbf{0}}, \Omega_{C_{\mathcal{B}}(X), \mathbf{0}}\right) \leqslant \mathrm{II} G_{1}\left(\mathcal{D}_{C_{\mathcal{A}}(X)}, \Omega_{C_{\mathcal{B}}(X), \mathbf{0}}\right)$. As $\mathcal{D}_{C_{\mathcal{A}}(X)} \subseteq \Omega_{C_{\mathcal{A}}(X), \mathbf{0}}$, this is true. Simply have Two use the exact same counter-play or strategy.

For (iii), define

- $\overleftarrow{T}_{\mathrm{I}, n}: \mathcal{O}(X, \mathcal{A}) \rightarrow \mathcal{D}_{C_{\mathcal{A}}(X)}$ by

$$
\overleftarrow{T}_{\mathrm{I}, n}(\mathcal{U})=\left\{f \in C_{\mathcal{A}}(X):(\exists U \in \mathcal{U})[f[X \backslash U]=1]\right\}
$$

- $\vec{T}_{\mathrm{II}, n}: C_{\mathcal{A}}(X) \times \mathcal{O}(X, \mathcal{A}) \rightarrow \mathscr{T}_{X}$ by $\vec{T}_{\mathrm{II}, n}(f, \mathcal{U})=U$, where $U \in \mathcal{U}$ is such that $f[X \backslash U]=\{1\}$ (if possible, otherwise set $\vec{T}_{\mathrm{II}, n}(f, \mathcal{U})=X$ ).

First check that the functions are well-defined. To see that $\overleftarrow{T}_{\mathrm{I}, n}(\mathcal{U})$ is a dense set in $C_{\mathcal{A}}(X)$ consider a basic open set $[f ; A, \varepsilon]$. Since $\mathcal{U} \in \mathcal{O}(X, \mathcal{A})$, there is a $U \in \mathcal{U}$ so that $A \subseteq U$. Since $X$ is $\mathcal{A}$-normal, we can find a continuous function $g: X \rightarrow[0,1]$ so that $g[A]=0$ and $g[X \backslash A]=1$. Define $h=f(1-g)+g$. Then $h\left\lceil_{A}=f, h[X \backslash U]=1\right.$. So $h \in[f ; A, \varepsilon] \cap \overleftarrow{T}_{\mathrm{I}, n}(\mathcal{U}, n)$. This shows that $\overleftarrow{T}_{\mathrm{I}, n}(\mathcal{U}, n)$ is dense. It is clear that $\vec{T}_{\mathrm{II}, n}$ maps into the appropriate space.

We next check $(\operatorname{Tr} 1)$. Suppose $f \in \overleftarrow{T}_{\mathrm{I}, n}(\mathcal{U})$. We need to check that $\vec{T}_{\mathrm{II}, n}(f, \mathcal{U}) \in \mathcal{U}$. Because $f \in \overleftarrow{T}_{\mathrm{I}, n}(\mathcal{U})$, we can find a $U \in \mathcal{U}$ so that $f[X \backslash U]=\{1\}$. Thus $\vec{T}_{\mathrm{II}, n}(f, \mathcal{U})=U \in \mathcal{U}$

Now we check (Tr2), that is, that the $\vec{T}_{\text {II }, n}$ translate from $\Omega_{C_{\mathcal{B}}(X), \mathbf{0}}$ to $\Lambda(X, \mathcal{B})$. Suppose $f_{n} \in \overleftarrow{T}_{\mathrm{I}, n}\left(\mathcal{U}_{n}\right)$ and

$$
\left\{f_{n}: n \in \omega\right\} \in \Omega_{C_{\mathcal{B}}(X), \mathbf{0}} .
$$

We need to see that $\left\{\vec{T}_{\text {II }, n}\left(f_{n}, \mathcal{U}_{n}\right): n \in \omega\right\} \in \Lambda(X, \mathcal{B})$. Notice $\vec{T}_{\text {II }, n}\left(f_{n}, \mathcal{U}_{n}\right)=U_{n} \in \mathcal{U}_{n}$ with the property that $f_{n}\left[X \backslash U_{n}\right]=1$. Let $B \in \mathcal{B}$. Then there is an $n_{0}$ so that $f_{n_{0}} \in[\mathbf{0} ; B, 1]$. Thus $B \subseteq f_{n_{0}}^{-1}[(-1,1)]$, and so $B \cap\left(X \backslash U_{n_{0}}\right)=\emptyset$. Therefore $B \subseteq U_{n_{0}}$. There is an $n_{1}>n_{0}$ so that $f_{n_{1}} \in[\mathbf{0} ; B, 1] \backslash\left\{f_{k}: k \leqslant n_{0}\right\}$ and so $B \subseteq U_{n_{1}}$. Continuing this process inductively, we see that $B$ is covered infinitely many times and that $\left\{U_{n}: n \in \omega\right\} \in \Lambda(X, \mathcal{B})$.

Corollary 15. Let $X$ be a Tychonoff space and $\mathcal{A}, \mathcal{B} \subseteq \wp(X)$. Then

(i) $G_{1}\left(\mathscr{N}_{C_{\mathcal{A}(X)}}(\mathbf{0}), \neg \Omega_{C_{\mathcal{B}(X)}, \mathbf{0}}\right) \leqslant{ }_{\text {II }} G_{1}(\mathscr{N}[\mathcal{A}], \neg \Lambda(X, \mathcal{B}))$

(ii) $G_{1}\left(\mathscr{T}_{C_{\mathcal{A}(X)}}, \neg \Omega_{C_{\mathcal{B}(X)}, \mathbf{0}}\right) \leqslant \mathrm{II}_{1} G_{1}\left(\mathscr{N}_{C_{\mathcal{A}(X)}}(\mathbf{0}), \neg \Omega_{C_{\mathcal{B}(X)}, \mathbf{0}}\right)$

(iii) $G_{1}\left(\mathscr{T}_{C_{\mathcal{A}}(X)}, \mathrm{CD}_{C_{\mathcal{B}}(X)}\right) \leqslant{ }_{\mathrm{II}} G_{1}\left(\mathscr{T}_{C_{\mathcal{A}(X)}}, \neg \Omega_{C_{\mathcal{B}(X)}, \mathbf{0}}\right)$

(iv) If $\mathcal{A}$ consists of closed sets, $X$ is $\mathcal{A}$-normal, and $\mathcal{B}$ consists of $\mathbb{R}$-bounded sets, then

$$
G_{1}(\mathscr{N}[\mathcal{A}], \neg \Lambda(X, \mathcal{B})) \leqslant{ }_{\mathrm{II}} G_{1}\left(\mathscr{T}_{C_{\mathcal{A}}(X)}, \mathrm{CD}_{C_{\mathcal{B}}(X)}\right) .
$$


Thus if $\mathcal{A}$ consists of closed sets, $X$ is $\mathcal{A}$-normal, and $\mathcal{B}$ consists of $\mathbb{R}$-bounded sets, then all these games are equivalent.

Proof. First we check that $G_{1}\left(\mathscr{N}_{C_{\mathcal{A}(X)}}(\mathbf{0}), \neg \Omega_{C_{\mathcal{B}(X)}, \mathbf{0}}\right) \leqslant_{\text {II }} G_{1}(\mathscr{N}[\mathcal{A}], \neg \Lambda(X, \mathcal{B}))$. Define

- $\overleftarrow{T}_{\mathrm{I}, n}: \mathscr{N}[\mathcal{A}] \rightarrow \mathscr{N}_{C_{\mathcal{A}(X)}}(\mathbf{0})$ by

$$
\overleftarrow{T}_{\mathrm{I}, n}(\mathscr{N}(A))=\left[\mathbf{0} ; A, 2^{-n}\right]
$$

- $\vec{T}_{\mathrm{II}, n}: C_{\mathcal{A}}(X) \times \mathscr{N}[\mathcal{A}] \rightarrow \mathscr{T}_{X}$ by $\vec{T}_{\mathrm{II}, n}(f, \mathscr{N}(A))=f^{-1}\left[\left(-2^{-n}, 2^{-n}\right)\right]$.

The maps are well-defined since the continuous pre-image of an open set is open.

We check (Tr1). Suppose $f \in \overleftarrow{T}_{\mathrm{I}, n}(\mathscr{N}(A))$. We need to check that $\vec{T}_{\mathrm{II}, n}(f, \mathscr{N}(A)) \in \mathscr{N}(A)$ i.e. that $A \subseteq f^{-1}\left[\left(-2^{-n}, 2^{-n}\right)\right]$. Since $f \in \overleftarrow{T}_{\mathrm{I}, n}(\mathscr{N}(A))=\left[\mathbf{0} ; A, 2^{-n}\right], f[A] \subseteq\left(-2^{-n}, 2^{-n}\right)$. Thus $A \subseteq f^{-1}\left[\left(-2^{-n}, 2^{-n}\right)\right]$.

We check (Tr2). Suppose $f_{n} \in \overleftarrow{T}_{\mathrm{I}, n}\left(\mathscr{N}\left(A_{n}\right)\right)$ and that $\left\{f_{n}: n \in \omega\right\} \notin \Omega_{C_{\mathcal{B}(X)}, \mathbf{0}}$. Then $f_{n} \in\left[\mathbf{0} ; A_{n}, 2^{-n}\right]$ and there is a $B \in \mathcal{B}$, an $\varepsilon>0$, and an $N \in \omega$ so that for all $n \geqslant N, f_{n} \notin[\mathbf{0} ; B, \varepsilon]$. We need to show that $\left\{f_{n}^{-1}\left[\left(-2^{-n}, 2^{-n}\right)\right]: n \in \omega\right\} \notin \Lambda(X, \mathcal{B})$. We proceed by way of contradiction. Suppose in particular that there is a $n \geqslant N$ so that $2^{-n}<\varepsilon$ and $B \subseteq f_{n}^{-1}\left[\left(-2^{-n}, 2^{-n}\right)\right]$. Then $f_{n} \in\left[\mathbf{0} ; B, 2^{-n}\right] \subseteq[\mathbf{0} ; B, \varepsilon]$. This is a contradiction.

Then $G_{1}\left(\mathscr{T}_{C_{\mathcal{A}(X)}}(\mathbf{0}), \neg \Omega_{C_{\mathcal{B}(X)}, \mathbf{0}}\right) \leqslant \mathrm{II} G_{1}\left(\mathscr{N}_{C_{\mathcal{A}(X)}}(\mathbf{0}), \neg \Omega_{C_{\mathcal{B}(X)}, \mathbf{0}}\right)$ is true as $\mathscr{N}_{C_{\mathcal{A}(X)}}(\mathbf{0}) \subseteq \mathscr{T}_{C_{\mathcal{A}(X)}}$.

To see that $G_{1}\left(\mathscr{T}_{C_{\mathcal{A}}(X)}, \mathrm{CD}_{C_{\mathcal{B}}(X)}\right) \leqslant \mathrm{II} G_{1}\left(\mathscr{T}_{C_{\mathcal{A}(X)}}, \neg \Omega_{C_{\mathcal{B}(X)}, \mathbf{0}}\right)$, observe that if Two can create a closed discrete set in response to player One, then Two has avoided having $\mathbf{0}$ as a cluster point.

Suppose $X$ is $\mathcal{A}$-normal and $\mathcal{B}$ consists of $\mathbb{R}$-bounded sets. For $U \in \mathscr{T}_{C_{\mathcal{A}}(X)}, V \in \mathscr{N}\left(A_{U}\right)$, and $n \in \omega$, identify a function $f_{U, V, n}: X \rightarrow \mathbb{R}$ with the property that $f_{U, V, n}\left\lceil A_{U}=f_{U}\right.$ and $f_{U, V, n}[X \backslash V]=\{n\}$. Such a function exists for the following reason. Since $X$ is $\mathcal{A}$-normal, there is a function $g$ so that $g\left[A_{U}\right]=0$ and $g[X \backslash V]=1$. Let $f_{U, V, n}=f_{U} \cdot(1-g)+n \cdot g$ and notice that $f_{U, V, n}$ is as required. Define

- $\overleftarrow{T}_{\mathrm{I}, n}: \mathscr{T}_{C_{\mathcal{A}}(X)} \rightarrow \mathscr{N}[\mathcal{A}]$ by $\overleftarrow{T}_{\mathrm{I}, n}(U)=\mathscr{N}\left(A_{U}\right)$

- $\vec{T}_{\mathrm{II}, n}: \mathscr{T}_{X} \times \mathscr{T}_{C_{\mathcal{A}}(X)} \rightarrow C_{\mathcal{A}}(X)$ by $\vec{T}_{\mathrm{II}, n}(V, U)=f_{U, V, n}$ (if possible, otherwise declare $\left.\vec{T}_{\mathrm{II}, n}(V, U)=\mathbf{0}\right)$.

We check $(\operatorname{Tr} 1)$. Suppose $V \in \overleftarrow{T}_{\mathrm{I}, n}(U)=\mathscr{N}\left(A_{U}\right)$. We need to check that $\vec{T}_{\mathrm{II}, n}(V, U)=$ $f_{U, V, n} \in U$. Since $V \in \mathscr{N}\left(A_{U}\right), f_{U, V, n}$ was chosen so that $f_{U, V, n} \uparrow_{A_{U}}=f_{U}$ which implies that $f_{U, V, n} \in U$.

We check (Tr2). Suppose $V_{n} \in \overleftarrow{T}_{\mathrm{I}, n}\left(U_{n}\right)=\mathscr{N}\left(A_{n}\right)$, where $A_{n}=A_{U_{n}}$ and $\left\{V_{n}: n \in \omega\right\} \notin$ $\Lambda(X, \mathcal{B})$. Then there is a $B \in \mathcal{B}$ and $N$ so that for all $n \geqslant N, B \nsubseteq V_{n}$. Say $\vec{T}_{\mathrm{II}, n}\left(V_{n}, U_{n}\right)=g_{n}$ and that $f_{U_{n}}=f_{n}$. Then $g_{n}\left\lceil A_{n}=f_{n}\right.$ and $g_{n}\left[X \backslash V_{n}\right]=\{n\}$. We proceed by way of contradiction. Let $f \in C_{\mathcal{B}}(X)$ be so that for all $n$, there is a $k \geqslant \max \{N, n\}$ so that $g_{k} \in\left[f ; B, 2^{-n}\right]$. Since $B \nsubseteq V_{k}$, there is an $x_{k} \in B \backslash V_{k}$. Thus $\left|g_{k}\left(x_{n}\right)-f\left(x_{n}\right)\right| \leqslant 2^{-n}$, and so $f\left(x_{n}\right) \geqslant k-1$. Proceeding in this way, we can produce an unbounded sequence $k_{n}$ and a collection of points $x_{n} \in B$ so that $f\left(x_{n}\right) \geqslant k_{n}-1$. But then $f$ is a continuous function where $f[B]$ is unbounded. So $B$ is not $\mathbb{R}$-bounded, which is a contradiction.

Corollary 16. Let $X$ be a Tychonoff space and $\mathcal{A}, \mathcal{B} \subseteq \wp(X)$. Then

(i) $G_{1}\left(\mathscr{N}_{C_{\mathcal{A}(X)}}(\mathbf{0}), \neg \Gamma_{C_{\mathcal{B}(X)}, \mathbf{0}}\right) \leqslant{ }_{\mathrm{II}} G_{1}(\mathscr{N}[\mathcal{A}], \neg \Gamma(X, \mathcal{B}))$ 
(ii) $G_{1}\left(\mathscr{N}_{C_{\mathcal{A}(X)}}(\mathbf{0}), \neg \Omega_{C_{\mathcal{B}(X)}, \mathbf{0}}\right) \leqslant{ }_{\mathrm{II}} G_{1}\left(\mathscr{N}_{C_{\mathcal{A}(X)}}(\mathbf{0}), \neg \Gamma_{C_{\mathcal{B}(X)}, \mathbf{0}}\right)$

Proof. Part (i) of this corollary is essentially the same as (i) of Corollary 15.

To see that $G_{1}\left(\mathscr{N}_{C_{\mathcal{A}(X)}}(\mathbf{0}), \neg \Omega_{C_{\mathcal{B}(X)}, \mathbf{0}}\right) \leqslant \mathrm{II}_{1} G_{1}\left(\mathscr{N}_{C_{\mathcal{A}(X)}}(\mathbf{0}), \neg \Gamma_{C_{\mathcal{B}(X)}, \mathbf{0}}\right)$, simply notice that if Two can avoid clustering around $\mathbf{0}$, then Two can certainly avoid converging to $\mathbf{0}$.

Definition 18. For a collection $\mathcal{A}$, we say that $\mathcal{B} \subseteq \mathcal{A}$ is a selection basis for $\mathcal{A}$ if

$$
(\forall A \in \mathcal{A})(\exists B \in \mathcal{B})(B \subseteq A) .
$$

Definition 19. For collections $\mathcal{A}$ and $\mathcal{R}$, we say that $\mathcal{R}$ is a reflection of $\mathcal{A}$ if

$$
\{\operatorname{ran}(f): f \in \operatorname{choice}(\mathcal{R})\}
$$

is a selection basis for $\mathcal{A}$.

Theorem 17. [2, Corollary 17] If $\mathcal{R}$ is a reflection of $\mathcal{A}$, then $G_{1}(\mathcal{A}, \mathcal{B})$ and $G_{1}(\mathcal{R}, \neg \mathcal{B})$ are dual.

Corollary 18. [1, Corollary 21] For any collection $\mathcal{A}$ of subsets of a space $X$ and any collection $\mathcal{B}$, the games $G_{1}(\mathcal{O}(X, \mathcal{A}), \mathcal{B})$ and $G_{1}(\mathscr{N}[\mathcal{A}], \neg \mathcal{B})$ are dual.

Proposition 19. Suppose $X$ is a topological space, $x \in X$, and $\mathcal{B} \subseteq \wp(X)$. Then $G_{1}\left(\Omega_{X, x}, \mathcal{B}\right)$ and $G_{1}(\mathscr{N}(x), \neg \mathcal{B})$ are dual.

Proof. It suffices to show that

$$
\{\operatorname{ran}(C): C \in \operatorname{choice}(\mathscr{N}(x))\} \subseteq \Omega_{X, x}
$$

and is a selection basis for $\Omega_{X, x}$. Clearly, each $\operatorname{ran}(C) \in \Omega_{X, x}$. Now let $F \in \Omega_{X, x}$. Then for each $U \in \mathscr{N}(x)$, there is an $x_{U} \in F \cap U$. Define a choice function $C$ for $\mathscr{N}(x)$ by $C(U)=x_{U}$. Notice that

$$
\operatorname{ran}(C)=\left\{x_{U}: U \in \mathscr{N}(x)\right\} \subseteq F
$$

Corollary 20. $G_{1}\left(\Omega_{C_{\mathcal{A}}(X), \mathbf{0}}, \Omega_{C_{\mathcal{B}}(X), \mathbf{0}}\right)$ and $G_{1}\left(\mathscr{N}_{C_{\mathcal{A}}(X)}(\mathbf{0}), \neg \Omega_{C_{\mathcal{B}}(X), \mathbf{0}}\right)$ are dual.

Proposition 21. $G_{1}\left(\mathcal{D}_{C_{\mathcal{A}}(X)}, \Omega_{C_{\mathcal{B}}(X), \mathbf{0}}\right)$ and $G_{1}\left(\mathscr{T}_{C_{\mathcal{A}}(X)}, \neg \Omega_{C_{\mathcal{B}}(X), \mathbf{0}}\right)$ are dual. Therefore whenever II $\uparrow G_{1}\left(\mathscr{T}_{C_{\mathcal{A}}(X)}, \mathrm{CD}_{C_{\mathcal{B}}(X)}\right)$, we have that I $\uparrow G_{1}\left(\mathcal{D}_{C_{\mathcal{A}}(X)}, \Omega_{C_{\mathcal{B}}(X), \mathbf{0}}\right)$. This is also true for going from Markov strategies to pre-determined strategies.

Proof. We can use reflection to show that $G_{1}\left(\mathcal{D}_{C_{\mathcal{A}}(X)}, \Omega_{C_{\mathcal{B}}(X), \mathbf{0}}\right)$ and $G_{1}\left(\mathscr{T}_{C_{\mathcal{A}}(X)}, \neg \Omega_{C_{\mathcal{B}}(X), \mathbf{0}}\right)$ are dual and that $G_{1}\left(\mathcal{D}_{C_{\mathcal{A}}(X)}, \neg \mathrm{CD}_{C_{\mathcal{B}}(X)}\right)$ and $G_{1}\left(\mathscr{T}_{C_{\mathcal{A}}(X)}, \mathrm{CD}_{C_{\mathcal{B}}(X)}\right)$ are dual as well. First check that

$$
\left\{\operatorname{ran}(C): C \in \operatorname{choice}\left(\mathscr{T}_{C_{\mathcal{A}}(X)}\right)\right\} \subseteq \mathcal{D}_{C_{\mathcal{A}}(X)}
$$

and is a selection basis for $\mathcal{D}_{C_{\mathcal{A}}(X)}$. Clearly, each $\operatorname{ran}(C) \in \mathcal{D}_{C_{\mathcal{A}}(X)}$. Now let $D \in \mathcal{D}_{C_{\mathcal{A}}(X)}$. Then for each $U \in \mathscr{T}_{C_{\mathcal{A}}(X)}$, there is an $f_{U} \in D \cap U$. Define a choice function $C$ for $\mathscr{T}_{C_{\mathcal{A}}(X)}$ by $C(U)=f_{U}$. Notice that

$$
\operatorname{ran}(C)=\left\{f_{U}: U \in \mathscr{T}_{C_{\mathcal{A}}(X)}\right\} \subseteq D
$$

Thus $G_{1}\left(\mathcal{D}_{C_{\mathcal{A}}(X)}, \mathcal{C}\right)$ and $G_{1}\left(\mathscr{T}_{C_{\mathcal{A}}(X)}, \neg \mathcal{C}\right)$ are dual for any $\mathcal{C} \subseteq \wp\left(C_{\mathcal{B}}(X)\right)$.

Therefore,

$$
\mathrm{II} \uparrow G_{1}\left(\mathscr{T}_{C_{\mathcal{A}}(X)}, \mathrm{CD}_{C_{\mathcal{B}}(X)}\right) \Longrightarrow \mathrm{II} \uparrow G_{1}\left(\mathscr{T}_{C_{\mathcal{A}}(X)}, \neg \Omega_{C_{\mathcal{B}}(X), \mathbf{0}}\right) \Longleftrightarrow \mathrm{I} \uparrow G_{1}\left(\mathcal{D}_{C_{\mathcal{A}}(X)}, \Omega_{C_{\mathcal{B}}(X), \mathbf{0}}\right) .
$$

The analogous results hold for Markov and pre-determined strategies. 


\section{Covering Properties}

Lemma 22. Suppose $X$ is a Tychonoff space. Then the following are equivalent:

(i) $\mathrm{I} \underset{\text { pre }}{\uparrow} G_{1}\left(\mathscr{N}_{C_{\mathcal{A}}(X)}(\mathbf{0}), \neg \Gamma_{C_{\mathcal{B}}(X), \mathbf{0}}\right)$,

(ii) $\mathrm{I} \underset{\text { pre }}{\uparrow} G_{1}\left(\mathscr{N}_{C_{\mathcal{A}}(X)}(\mathbf{0}), \neg \Omega_{C_{\mathcal{B}}(X), \mathbf{0}}\right)$,

(iii) $\operatorname{cof}\left(\mathscr{N}_{C_{\mathcal{A}}(X)}(\mathbf{0}) ; \mathscr{N}_{C_{\mathcal{B}}(X)}(\mathbf{0}), \supseteq\right)=\omega$.

Proof. Clearly, (i) implies (ii).

Suppose I $\uparrow G_{1}\left(\mathscr{N}_{C_{\mathcal{A}}(X)}(\mathbf{0}), \neg \Omega_{C_{\mathcal{B}}(X), \mathbf{0}}\right)$. Then we get a sequence of neighborhoods $\left[\mathbf{0} ; A_{n}, \varepsilon_{n}\right]$. Now, let $B \in \mathcal{B}, \varepsilon>0$, and consider $[\mathbf{0} ; B, \varepsilon]$. Suppose $\left[\mathbf{0} ; A_{n}, \varepsilon_{n}\right] \nsubseteq[\mathbf{0} ; B, \varepsilon]$ for any $n$. Then we have functions $f_{n} \in\left[\mathbf{0} ; A_{n}, \varepsilon_{n}\right] \backslash[\mathbf{0} ; B, \varepsilon]$. Consider the play $\left[\mathbf{0} ; A_{0}, \varepsilon_{0}\right], f_{0}, \cdots$ of the game $G_{1}\left(\mathscr{T}_{C_{\mathcal{A}}(X)}, \neg \Omega_{C_{\mathcal{B}}(X), \mathbf{0}}\right)$ according to the winning strategy. Because none of the $f_{n}$ are in $[\mathbf{0} ; B, \varepsilon]$, the $f_{n}$ fail to accumulate to $\mathbf{0}$ in $C_{\mathcal{B}}(X)$. This is a contradiction. So (ii) implies (iii).

Now let $U_{n}$ be a sequence of $C_{\mathcal{A}}(X)$ neighborhoods of $\mathbf{0}$ which is cofinal in the $C_{\mathcal{B}}(X)$ neighborhoods. We can assume without loss of generality that the $U_{n}$ are descending. Define a strategy $\sigma$ for player One in $G_{1}\left(\mathscr{N}_{C_{\mathcal{A}}(X)}(\mathbf{0}), \neg \Gamma_{C_{\mathcal{B}}(X), \mathbf{0}}\right)$ by $\sigma(n)=U_{n}$. Suppose that $f_{n} \in U_{n}$ for all $n$. Let $[\mathbf{0} ; B, \varepsilon]$ be an arbitrary $C_{\mathcal{B}}(X)$-nhood of $\mathbf{0}$. Then there is an $N$ so that for all $n \geqslant N, U_{n} \subseteq[\mathbf{0} ; B, \varepsilon]$. Thus for all $n \geqslant N, f_{n} \in[\mathbf{0} ; B, \varepsilon]$. So $f_{n} \rightarrow \mathbf{0}$ in $C_{\mathcal{B}}(X)$. Therefore (iii) implies (i).

The following generalizes V.416 from [12, p. 460]. Moreover, if we replace $\mathcal{A}$ with the space of singletons $X$, we obtain Theorem 1 of Gerlits and Nagy, [6].

Lemma 23. Assume $\mathcal{A}, \mathcal{B} \subseteq \wp(X)$. Then $\mathrm{I} \underset{\text { pre }}{\uparrow} G_{1}(\mathscr{N}[\mathcal{A}], \neg \mathcal{O}(X, \mathcal{B}))$ if and only if $\operatorname{cof}(\mathcal{A} ; \mathcal{B}, \subseteq) \leqslant \omega$.

Proof. Suppose I $\uparrow G_{1}(\mathscr{N}[\mathcal{A}], \neg \mathcal{O}(X, \mathcal{B}))$. Let $\sigma$ be an example of a pre-determined strategy for One in this game. Say $\operatorname{ran}(\sigma)=\left\{\mathscr{N}\left(A_{n}\right): n \in \omega\right\}$. We claim that $\left\{A_{n}: n \in \omega\right\}$ is cofinal for $\mathcal{B}$. Towards a contradiction suppose that there were an $B \in \mathcal{B}$ so that $B \nsubseteq A_{n}$ for all $n$. Then for each $n$, we can choose $x_{n} \in B \backslash A_{n}$. Then the sequence $\mathscr{N}\left(A_{0}\right), X \backslash\left\{x_{0}\right\}, \cdots$ would be a play of $G_{1}(\mathscr{N}[\mathcal{A}], \neg \mathcal{O}(X, \mathcal{B}))$. Since $\sigma$ is winning, $B \subseteq X \backslash\left\{x_{n}\right\}$ for some $n$. But then $x_{n} \in X \backslash\left\{x_{n}\right\}$, a contradiction. Therefore $\left\{A_{n}: n \in \omega\right\}$ is cofinal for $\mathcal{B}$ and $\operatorname{cof}(\mathcal{A} ; \mathcal{B}, \subseteq) \leqslant \omega$.

$\operatorname{Suppose} \operatorname{cof}(\mathcal{A} ; \mathcal{B}, \subseteq)=\omega$. Let $\left\{A_{n}: n \in \omega\right\}$ witness this. Define a strategy $\sigma$ by $\sigma(n)=\mathscr{N}\left(A_{n}\right)$. Now suppose $\sigma(0), U_{0}, \cdots$ is a play of $G_{1}(\mathscr{N}[\mathcal{A}], \neg \mathcal{O}(X, \mathcal{B}))$ according to $\sigma$. Let $B \in \mathcal{B}$. Then there is an $n$ so that $B \subseteq A_{n} \subseteq U_{n}$. Thus $\left\{U_{n}: n \in \omega\right\} \in \mathcal{O}(X, \mathcal{B})$ and $\sigma$ is winning.

The following generalizes a result of Telgársky [11] and extends Theorem 27 of [1].

Lemma 24. Assume $\mathcal{A}, \mathcal{B} \subseteq \wp(X)$, and $\mathcal{A}$ is a collection of $G_{\delta}$ sets. Then the following are equivalent:

(i) $\mathrm{I} \uparrow G_{1}(\mathscr{N}[\mathcal{A}], \neg \mathcal{O}(X, \mathcal{B}))$

(ii) $\operatorname{cof}(\mathcal{A} ; \mathcal{B}, \subseteq) \leqslant \omega$

(iii) $\mathrm{I} \underset{\text { pre }}{\uparrow} G_{1}(\mathscr{N}[\mathcal{A}], \neg \mathcal{O}(X, \mathcal{B}))$ 
Proof. Let $\sigma$ be a strategy for One. Without loss of generality, One is playing sets from $\mathcal{A}$ and Two plays open sets which contain One's play. For every $A \in \mathcal{A}$, let $\mathcal{U}_{A}$ be a countable collection of open sets so that $A=\bigcap \mathcal{U}_{A}$.

Define a tree in the following way. Let $T_{0}=\emptyset$. For $n \in \omega$, we define

$$
T_{n+1}=\left\{w \frown\langle\sigma(w), U\rangle: w \in T_{n} \text { and } U \in \mathcal{U}_{\sigma(w)}\right\} .
$$

Observe that each $T_{n}$ is countable as each $\mathcal{U}_{A}$ is countable. Hence,

$$
\mathscr{F}:=\bigcup_{n \in \omega}\left\{\sigma(w): w \in T_{n}\right\}
$$

is a countable subset of $\mathcal{A}$.

By way of contradiction, suppose there is some $B \in \mathcal{B}$ so that $B \nsubseteq A$ for all $A \in \mathscr{F}$. Let $A_{0}=\sigma(\emptyset)$. Since $B \nsubseteq A_{0}$, there must be some $x_{0} \in B \backslash A_{0}$. As $A_{0}=\bigcap \mathcal{U}_{A_{0}}$, there is some $U_{0} \in \mathcal{U}_{A_{0}}$ so that $A_{0} \subseteq U_{0}$ and $x_{0} \notin U_{0}$.

Recursively, this defines a run of the game $A_{0}, U_{0}, A_{1}, U_{1}, \ldots$ according to $\sigma$. So we can conclude that $\left\{U_{n}: n \in \omega\right\} \in \mathcal{O}(X, \mathcal{B})$. Thus, $B \subseteq U_{n}$ for some $n \in \omega$ but then $x_{n} \in U_{n}$, a contradiction. Therefore, $\operatorname{cof}(\mathcal{A} ; \mathcal{B}, \subseteq) \leqslant \omega$.

The rest of the equivalence is clear.

Note 1. Let $X$ be the one-point Lindelöfication of $\omega_{1}$ and consider $G_{1}\left(\mathscr{N}\left[[X]^{<\omega}\right], \neg \mathcal{O}\left(X,[X]^{<\omega}\right)\right)$. In $X,\left\{\omega_{1}\right\}$ is closed, but not a $G_{\delta}$. One has a winning strategy in $G_{1}\left(\mathscr{N}\left[[X]^{<\omega]}, \neg \mathcal{O}\left(X,[X]^{<\omega}\right)\right)\right.$, but $\operatorname{cof}\left([X]^{<\omega} ;[X]^{<\omega}, \subseteq\right)=\omega_{1}$.

Now consider $X=\mathbb{R}$. Let $\mathcal{M}$ be the meager subsets of $\mathbb{R}$. Then player One has a winning tactic (in two moves) for $G_{1}\left(\mathscr{N}[\mathcal{M}], \neg \mathcal{O}_{X}\right)$, but $\operatorname{cof}(\mathcal{M} ; X, \subseteq)=\operatorname{cov}(\mathcal{M})>\omega$.

\section{The Main Theorems}

Theorem 25. Suppose $X$ is a Tychonoff space and $\mathcal{A}, \mathcal{B} \subseteq \wp(X)$. Suppose $\mathcal{A}$ and $\mathcal{B}$ are idealbases and that $\mathcal{A}$ consists of closed sets. Then the following diagrams are true, where dashed arrows require the assumption that $X$ is $\mathcal{A}$-normal and dotted lines require the assumption that $\mathcal{B}$ consists of $\mathbb{R}$-bounded sets.

If $X$ is $\mathcal{A}$-normal, $\mathcal{B}$ consists of $\mathbb{R}$-bounded sets, and $\mathcal{A}$ consists of $G_{\delta}$ sets, then all of the statements across both diagrams are equivalent. 


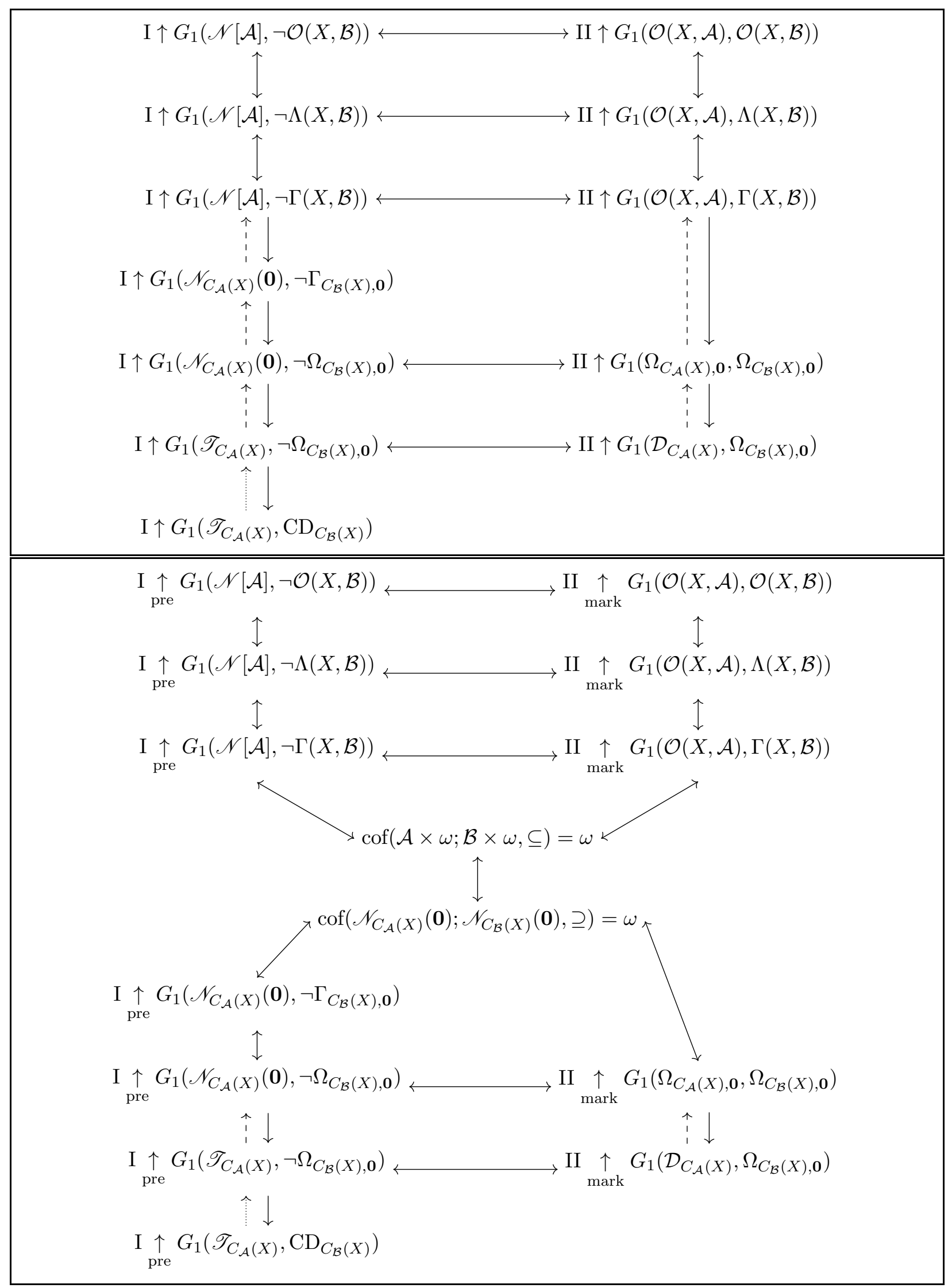


Proof. Since we have assumed that $\mathcal{A}$ and $\mathcal{B}$ are ideal-bases, Lemma 11 implies that all three versions of the generalized point-open game are equivalent for player One. This applies for full strategies and pre-determined strategies.

The fact that $\mathrm{I} \uparrow G_{1}(\mathscr{N}[\mathcal{A}], \neg \Psi(X, \mathcal{B}))$ is equivalent to $\mathrm{II} \uparrow G_{1}(\mathcal{O}(X, \mathcal{A}), \Psi(X, \mathcal{B})$ ) (where $\Psi$ is $\mathcal{O}, \Lambda$, or $\Gamma$ ) comes from the general reflection result from Clontz, Theorem 17. This also implies the analogous statements for pre-determined and Markov strategies. Since all of the versions of the generalized point-open game are equivalent for player One, we can conclude that all of the versions of the generalized Rothberger game are equivalent for player Two.

By Corollary 20, II $\uparrow G_{1}\left(\Omega_{C_{\mathcal{A}}(X), \mathbf{0}}, \Omega_{C_{\mathcal{B}}(X), \mathbf{0}}\right)$ if and only if I $\uparrow G_{1}\left(\mathscr{N}_{C_{\mathcal{A}}(X)}(\mathbf{0}), \neg \Omega_{C_{\mathcal{B}}(X), \mathbf{0}}\right)$, and also at the level of Markov/pre-determined strategies. Likewise, Proposition 21 implies that II $\uparrow G_{1}\left(\mathcal{D}_{C_{\mathcal{A}}(X)}, \Omega_{C_{\mathcal{B}}(X), \mathbf{0}}\right)$ is equivalent to I $\uparrow G_{1}\left(\mathscr{T}_{C_{\mathcal{A}}(X)}, \neg \Omega_{C_{\mathcal{B}}(X), \mathbf{0}}\right)$, and also at the level of Markov/pre-determined strategies.

Corollary 14 yields the implications between $G_{1}(\mathcal{O}(X, \mathcal{A}), \Lambda(X, \mathcal{B})), G_{1}\left(\Omega_{C_{\mathcal{A}}(X), \mathbf{0}}, \Omega_{C_{\mathcal{B}}(X), \mathbf{0}}\right)$, and $G_{1}\left(\mathcal{D}_{C_{\mathcal{A}}(X)}, \Omega_{C_{\mathcal{B}}(X), \mathbf{0}}\right)$. Then Corollaries 15 and 16 provide the arrows between games for the rest of the left side of the diagram.

We now check the improved implications in the second diagram. By Lemma 23, we have that I $\underset{\text { pre }}{\uparrow} G_{1}(\mathscr{N}[\mathcal{A}], \neg \mathcal{O}(X, \mathcal{B}))$ if and only if $\operatorname{cof}(\mathcal{A} \times \omega, \mathcal{B} \times \omega)=\omega$. Then Lemma 3 implies that $\operatorname{cof}(\mathcal{A} \times \omega, \mathcal{B} \times \omega)=\omega$ if and only if $\operatorname{cof}\left(\mathscr{N}_{C_{\mathcal{A}}(X)}(\mathbf{0}) ; \mathscr{N}_{C_{\mathcal{B}}(X)}(\mathbf{0}), \supseteq\right)=\omega$. Finally, using Lemma 22 we see that $\operatorname{cof}\left(\mathscr{N}_{C_{\mathcal{A}}(X)}(\mathbf{0}) ; \mathscr{N}_{C_{\mathcal{B}}(X)}(\mathbf{0}), \supseteq\right)=\omega$ if and only if $\underset{\text { pre }}{\uparrow} G_{1}\left(\mathscr{N}_{C_{\mathcal{A}}(X)}(\mathbf{0}), \neg \Omega_{C_{\mathcal{B}}(X), \mathbf{0}}\right)$, which is in turn equivalent to $\mathrm{I} \underset{\text { pre }}{\uparrow} G_{1}\left(\mathscr{N}_{C_{\mathcal{A}}(X)}(\mathbf{0}), \neg \Gamma_{C_{\mathcal{B}}(X), \mathbf{0}}\right)$. This suffices to improve the arrows from the first diagram and finishes the second diagram.

Theorem 26. Suppose $X$ is a Tychonoff space and $\mathcal{A}, \mathcal{B} \subseteq \wp(X)$. Suppose $\mathcal{A}$ and $\mathcal{B}$ are idealbases and that $\mathcal{A}$ consists of closed sets. Then the following diagrams are true, where dashed arrows require the assumption that $X$ is $\mathcal{A}$-normal and dotted lines require the assumption that $X$ is $\mathcal{A}$-normal and $\mathcal{B}$ consists of $\mathbb{R}$-bounded sets.

If $X$ is $\mathcal{A}$-normal, $\mathcal{B}$ consists of $\mathbb{R}$-bounded sets, and $\mathcal{A} \prec \mathcal{B}$, then all of the statements across both diagrams are equivalent. Correction: If $\mathcal{A}=\mathcal{B}$ are the finite subsets of $X$, or $\mathcal{A}=\mathcal{B}$ are the compact subsets of $X$, then all of the statements across both diagrams are equivalent.

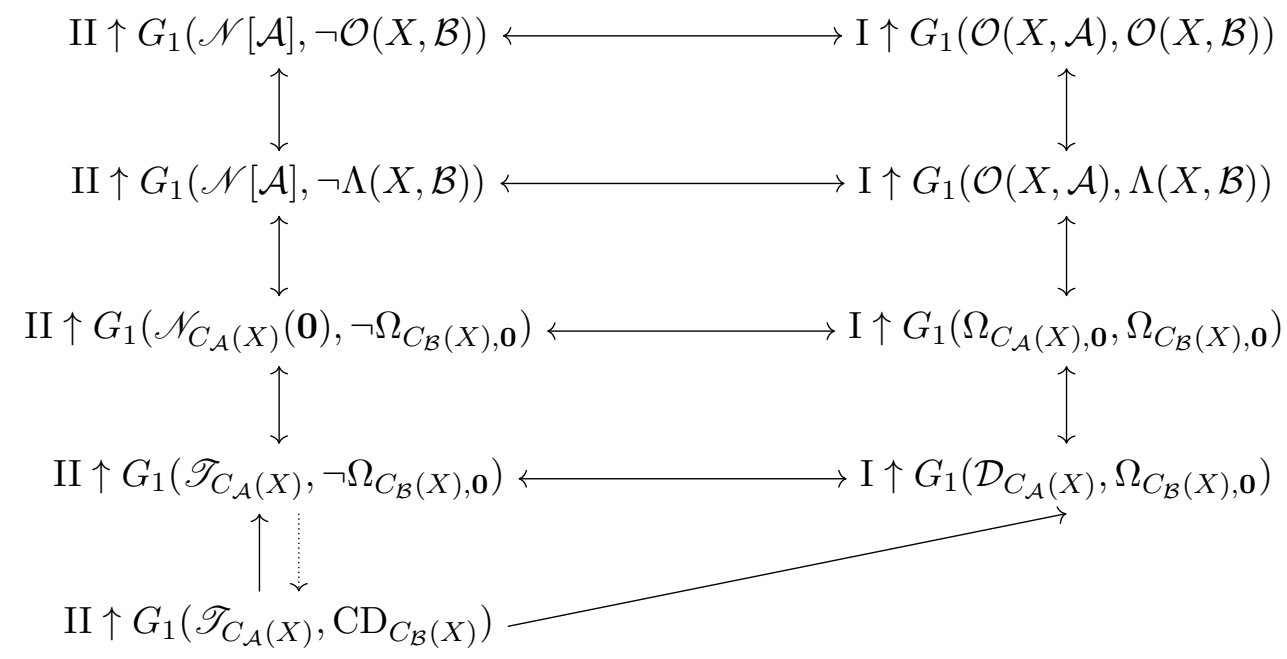




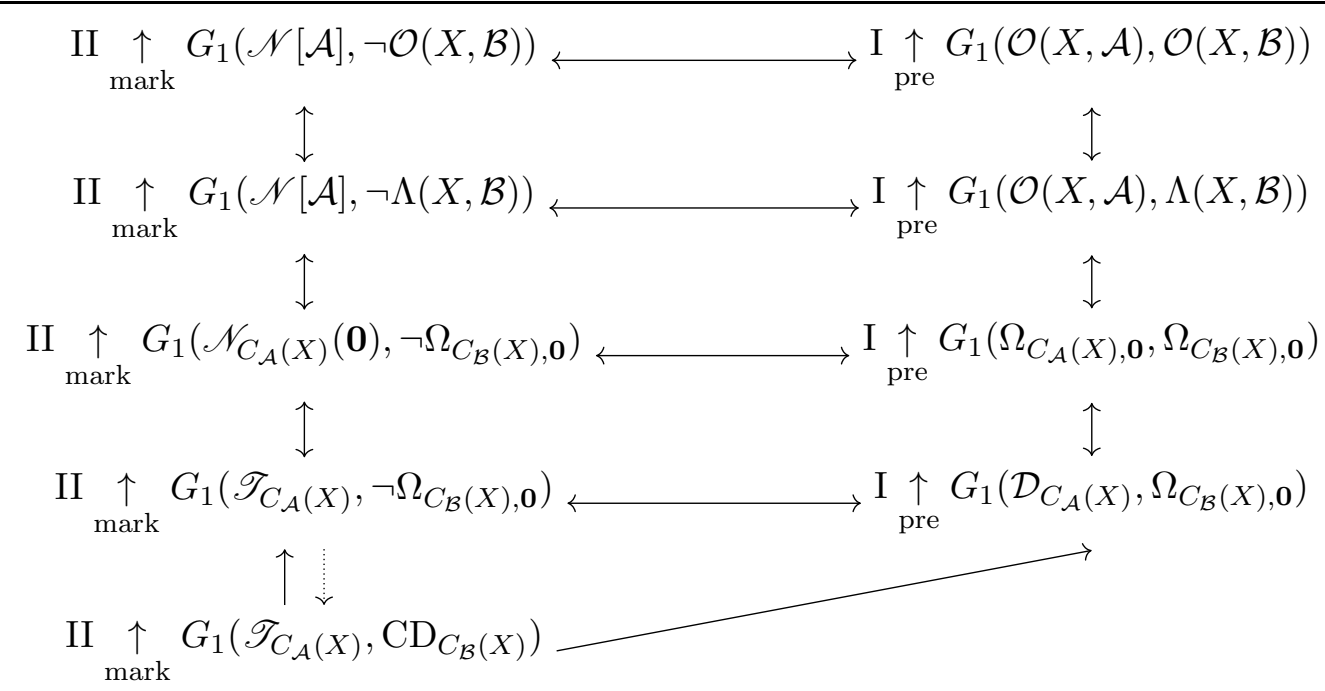

Proof. Since $\mathcal{A}$ and $\mathcal{B}$ are pre-ideals, the versions of the point-open game are equivalent.

The arrows between the versions of the point-open game and versions of the Rothberger game come from the duality of the point-open and Rothberger games. From this, the versions of the Rothberger game are equivalent.

Corollaries 15 and 16 generates arrows on the left side of the diagram. Similarly, Corollary 14 provides arrows on the right side of the diagram.

By Corollary, 20, I $\underset{\text { pre }}{\uparrow} G_{1}\left(\Omega_{C_{\mathcal{A}}(X), \mathbf{0}}, \Omega_{C_{\mathcal{B}}(X), \mathbf{0}}\right)$ if and only if II $\underset{\operatorname{mark}}{\uparrow} G_{1}\left(\mathscr{N}_{C_{\mathcal{A}}(X)}(\mathbf{0}), \neg \Omega_{C_{\mathcal{B}}(X), \mathbf{0}}\right)$.

Proposition 21 adds the implications from the statement II $\underset{\text { mark }}{\uparrow} G_{1}\left(\mathscr{T}_{C_{\mathcal{A}}(X)}, \mathrm{CD}_{C_{\mathcal{B}}(X)}\right)$ to I $\uparrow$ $G_{1}\left(\mathcal{D}_{C_{\mathcal{A}}(X)}, \Omega_{C_{\mathcal{B}}(X), \mathbf{0}}\right)$ and then to II $\underset{\text { mark }}{\uparrow} G_{1}\left(\mathscr{T}_{C_{\mathcal{A}}(X)}, \neg \Omega_{C_{\mathcal{B}}(X), \mathbf{0}}\right)$.

With these connections, the main block of the diagram becomes equivalent without any extra assumptions needed.

If $\mathcal{A} \prec \mathcal{B}$, Lemma 8 applies and all of the statements across the two diagrams are equivalent. Correction: If $\mathcal{A}=\mathcal{B}$ are either the finite or compact subsets of $X$, Lemma 8's revision in arXiv:2102.00296 applies and all of the statements across the two diagrams are equivalent.

Note 2. Suppose $\mathcal{A}=\mathcal{B}=[\mathbb{R}]^{\omega}$. Define a strategy for One in $G_{1}(\mathcal{O}(X, \mathcal{A}), \mathcal{O}(X, \mathcal{B}))$ as follows: In the $n^{\text {th }}$ inning, for any countable set $A \subseteq \mathbb{R}$, choose $U_{A, n}$ to be an open set so that $A \subseteq U_{A, n}$ and $U_{A, n}$ has Lebesgue measure $<2^{-n}$. Then $\sigma(n)=\left\{U_{A, n}: A \in \mathcal{A}\right\}$. This is a pre-determined winning strategy for One.

Consider a strategy for Two in $G_{1}\left(\mathcal{D}_{C_{\mathcal{A}}(X)}, \Omega_{C_{\mathcal{B}}(X), \mathbf{0}}\right)$ defined as follows: In the $n^{\text {th }}$ inning, One's play must have non-trivial intersection with $\left[\mathbf{0} ; \mathbb{Q}, 2^{-n}\right]$. Let Two choose $f_{n}$ in this intersection. Then as in the previous example, $f_{n} \rightarrow \mathbf{0}$. This shows that if $\mathcal{A}$ does not consist of closed sets, then the properties do not have to be equivalent.

Note 3. If we do not require that $\mathcal{A}$ be an ideal base, then the statements

- II $\uparrow G_{1}(\mathscr{N}[\mathcal{A}], \neg \Gamma(X, \mathcal{B}))$,

- I $\uparrow G_{1}(\mathcal{O}(X, \mathcal{A}), \Gamma(X, \mathcal{B}))$, and

- II $\uparrow G_{1}\left(\mathscr{N}_{C_{\mathcal{A}}(X)}(\mathbf{0}), \neg \Gamma_{C_{\mathcal{B}}(X), \mathbf{0}}\right)$ 
are all strictly weaker than any of those present in the first diagram of the previous theorem. This is also true for Markov/pre-determined strategies. The counter example of $X=\mathbb{Z}$ with $\mathcal{A}$ and $\mathcal{B}$ both set to be the singleton subsets of $\mathbb{Z}$ demonstrates this.

Assuming that $\mathcal{A}$ is an ideal base makes the situation more complicated. In that situation I $\uparrow G_{1}(\mathscr{N}[\mathcal{A}], \neg \mathcal{O}(X, \mathcal{B}))$ implies that I $\uparrow G_{1}(\mathscr{N}[\mathcal{A}], \neg \Gamma(X, \mathcal{B}))$. So to find a space $X$ where II $\ngtr G_{1}(\mathscr{N}[\mathcal{A}], \neg \mathcal{O}(X, \mathcal{B}))$ and II $\uparrow G_{1}(\mathscr{N}[\mathcal{A}], \neg \Gamma(X, \mathcal{B}))$, we need for $G_{1}(\mathscr{N}[\mathcal{A}], \neg \mathcal{O}(X, \mathcal{B}))$ to be undetermined and $X$ to not be a $\gamma$-set. These are necessary but not sufficient conditions. We do not currently know of any counter-examples, but we also do not know a good reason why the games should be equivalent for player Two.

\section{Applications}

Corollaries 27 and 28 are direct applications of Lemma 24.

Corollary 27. Suppose $X$ is a space where all closed sets are $G_{\delta}$ sets, $\mathcal{A}$ consists of the closed nowhere dense sets, and $\mathcal{B}$ is the set of all singleton subsets of $X$. Then One has a winning strategy in $G_{1}(\mathscr{N}[\mathcal{A}], \neg \mathcal{O}(X, \mathcal{B}))$ if and only if $X$ is meager.

Corollary 28. Suppose $X$ is a space, $\mathcal{A}$ consists of the $G_{\delta} \mu$-null sets with respect to a Borel measure $\mu$, and $\mathcal{B}$ is the set of all singleton subsets of $X$. Then One has a winning strategy in $G_{1}(\mathscr{N}[\mathcal{A}], \neg \mathcal{O}(X, \mathcal{B}))$ if and only if $X$ is $\mu$-null; i.e., $\mu$ is the trivial zero measure.

The following summarizes a majority of the results from [3].

Theorem 29. Suppose $X$ is a Tychonoff space. Then

(i) $G_{1}\left(\mathscr{N}\left[[X]^{<\omega}\right], \neg \Omega_{X}\right), G_{1}\left(\mathscr{N}_{C_{p}(X)}(\mathbf{0}), \neg \Omega_{C_{p}(X), \mathbf{0}}\right)$, and $G_{1}\left(\mathscr{T}_{C_{p}(X)}, \mathrm{CD}_{C_{p}(X)}\right)$ are equivalent,

(ii) $G_{1}\left(\Omega_{X}, \Omega_{X}\right), G_{1}\left(\Omega_{C_{p}(X), \mathbf{0}}, \Omega_{C_{p}(X), \mathbf{0}}\right)$, and $G_{1}\left(\mathcal{D}_{C_{p}(X)}, \Omega_{C_{p}(X), \mathbf{0}}\right)$ are equivalent,

(iii) The two groups of games in (i) and (ii) are dual to each other,

(iv) I $\uparrow G_{\text {pre }}\left(\mathscr{T}_{C_{p}(X)}, \mathrm{CD}_{C_{p}(X)}\right)$ iff $X$ is countable iff $C_{p}(X)$ is first countable,

(v) For player One, the games $G_{1}\left(\mathscr{N}\left[[X]^{<\omega}\right], \neg \Gamma_{X}\right)$ and $G_{1}\left(\mathscr{N}_{C_{p}(X)}(\mathbf{0}), \neg \Gamma_{C_{p}(X), \mathbf{0}}\right)$ are equivalent to $G_{1}\left(\mathscr{N}\left[[X]^{<\omega}\right], \neg \Omega_{X}\right)$ and $G_{1}\left(\mathscr{N}_{C_{p}(X)}(\mathbf{0}), \neg \Omega_{C_{p}(X), \mathbf{0}}\right)$,

(vi) For player Two, $G_{1}\left(\Omega_{X}, \Omega_{X}\right)$ and $G_{1}\left(\Omega_{X}, \Gamma_{X}\right)$ are equivalent,

(vii) $\mathrm{I} \uparrow G_{\text {pre }}\left(\Omega_{X}, \Omega_{X}\right)$ if and only if $\mathrm{I} \uparrow G_{1}\left(\Omega_{X}, \Omega_{X}\right)$.

The following summarizes a majority of the results from [1].

Theorem 30. Suppose $X$ is a Tychonoff space. Then

(i) $G_{1}\left(\mathscr{N}[K(X)], \neg \mathcal{K}_{X}\right), G_{1}\left(\mathscr{N}_{C_{k}(X)}(\mathbf{0}), \neg \Omega_{C_{k}(X), \mathbf{0}}\right)$, and $G_{1}\left(\mathscr{T}_{C_{k}(X)}, \mathrm{CD}_{C_{k}(X)}\right)$ are equivalent,

(ii) $G_{1}\left(\mathcal{K}_{X}, \mathcal{K}_{X}\right), G_{1}\left(\Omega_{C_{k}(X), \mathbf{0}}, \Omega_{C_{k}(X), \mathbf{0}}\right)$, and $G_{1}\left(\mathcal{D}_{C_{k}(X)}, \Omega_{C_{k}(X), \mathbf{0}}\right)$ are equivalent,

(iii) The two groups of games in (i) and (ii) are dual to each other,

(iv) I $\uparrow G_{1}\left(\mathscr{T}_{C_{k}(X)}, \mathrm{CD}_{C_{k}(X)}\right)$ iff $X$ is hemicompact iff $C_{k}(X)$ is first-countable, 
(v) For player One, $G_{1}\left(\mathscr{N}[K(X)], \neg \Gamma_{k}(X)\right)$ and $G_{1}\left(\mathscr{N}_{C_{k}(X)}(\mathbf{0}), \neg \Gamma_{C_{k}(X), \mathbf{0}}\right)$ are equivalent to $G_{1}\left(\mathscr{N}[K(X)], \neg \mathcal{K}_{X}\right)$ and $G_{1}\left(\mathscr{N}_{C_{k}(X)}(\mathbf{0}), \neg \Omega_{C_{k}(X), \mathbf{0}}\right)$,

(vi) For player Two, $G_{1}\left(\mathcal{K}_{X}, \mathcal{K}_{X}\right)$ and $G_{1}\left(\mathcal{K}_{X}, \Gamma_{k}(X)\right)$ are equivalent,

(vii) $\mathrm{I} \underset{\text { pre }}{\uparrow} G_{1}\left(\mathcal{K}_{X}, \mathcal{K}_{X}\right)$ if and only if $\mathrm{I} \uparrow G_{1}\left(\mathcal{K}_{X}, \mathcal{K}_{X}\right)$.

Notice that the property of being $\sigma$-compact lies in between being countable and being hemicompact. If we use the fact that Theorems 25 and 26 apply to pairs $\mathcal{A}$ and $\mathcal{B}$, then we can generate a setup which characterizes $\sigma$-compactness in way that is similar to Theorems 29 and 30 .

Theorem 31. Suppose $X$ is a Tychonoff space. Then

(i) $G_{1}\left(\mathscr{N}[K(X)], \neg \Omega_{X}\right), G_{1}\left(\mathscr{N}_{C_{k}(X)}(\mathbf{0}), \neg \Omega_{C_{p}(X), \mathbf{0}}\right)$, and $G_{1}\left(\mathscr{T}_{C_{k}(X)}, \mathrm{CD}_{C_{p}(X)}\right)$ are equivalent,

(ii) $G_{1}\left(\mathcal{K}_{X}, \Omega_{X}\right), G_{1}\left(\Omega_{C_{k}(X), \mathbf{0}}, \Omega_{C_{p}(X), \mathbf{0}}\right)$, and $G_{1}\left(\mathcal{D}_{C_{k}(X)}, \Omega_{C_{p}(X), \mathbf{0}}\right)$ are equivalent,

(iii) The two groups of games in (i) and (ii) are dual to each other,

(iv) $\mathrm{I} \underset{\text { pre }}{\uparrow} G_{1}\left(\mathscr{T}_{C_{k}(X)}, \mathrm{CD}_{C_{p}(X)}\right)$ iff $X$ is $\sigma$-compact iff $\operatorname{cof}\left(\mathscr{N}_{C_{k}(X)}(\mathbf{0}) ; \mathscr{N}_{C_{p}(X)}(\mathbf{0}), \supseteq\right)=\omega$,

(v) For player One, the games $G_{1}\left(\mathscr{N}[K(X)], \neg \Gamma_{X}\right)$ and $G_{1}\left(\mathscr{N}_{C_{k}(X)}(\mathbf{0}), \neg \Gamma_{C_{p}(X), \mathbf{0}}\right)$ are equivalent to $G_{1}\left(\mathscr{N}[K(X)], \neg \Omega_{X}\right)$ and $G_{1}\left(\mathscr{N}_{C_{k}(X)}(\mathbf{0}), \neg \Omega_{C_{p}(X), \mathbf{0}}\right)$, and

(vi) For player Two, $G_{1}\left(\mathcal{K}_{X}, \Omega_{X}\right)$ and $G_{1}\left(\mathcal{K}_{X}, \Gamma_{X}\right)$ are equivalent.

\section{Open Questions}

- Is there a topological characterization of the statement $\operatorname{cof}(\mathcal{A} ; \mathcal{B}, \leqslant) \leqslant_{T} \omega^{\omega}$ ?

- Does I $\uparrow G_{1}\left(\mathcal{K}_{X}, \Omega_{X}\right)$ imply $\mathrm{I} \underset{\text { pre }}{\uparrow} G_{1}\left(\mathcal{K}_{X}, \Omega_{X}\right)$ ?

- More broadly, to what extent can the Pawlikowski generalization presented here be further generalized?

- If $\mathcal{A}$ is an ideal base, are $G_{1}(\mathscr{N}[\mathcal{A}], \neg \Gamma(X, \mathcal{B}))$ and $G_{1}(\mathscr{N}[\mathcal{A}], \neg \mathcal{O}(X, \mathcal{B}))$ equivalent for player Two?

- Can the assumption that $\mathcal{B}$ consists of $\mathbb{R}$-bounded sets be removed from Theorems 25 and $26 ?$

- To what extent can the techniques in this paper be used to study more complex selection principles like the Hurewicz property or the $\alpha$-Fréchet properties?

\section{References}

[1] Christopher Caruvana and Jared Holshouser, Closed discrete selection in the compact open topology, Topology Proceedings 56 (2020), 25 - 55.

[2] Steven Clontz, Dual selection games, Topology and its Applications 272 (2020), 107056. 
[3] Steven Clontz and Jared Holshouser, Limited information strategies and discrete selectivity, Topology and its Applications 265 (2019), 106815.

[4] Fred Galvin, Indeterminacy of point-open games., Bull. Acad. Pol. Sci., Sér. Sci. Math. Astron. Phys. 26 (1978), 445-449.

[5] Paul Gartside and Ana Mamatelashvili, The Tukey Order and Subsets of $\omega_{1}$, Order 35 (2018), no. 1, 139-155.

[6] J. Gerlits and Zs. Nagy, Some properties of $C(X)$, I, Topology and its Applications 14 (1982), no. $2,151-161$.

[7] Lj.D.R. Kočinac, Selected results on selection principles, Proceedings of the Third Seminar on Geometry and Topology (Tabriz, Iran), July 15-17, 2004, pp. 71-104.

[8] Ernest A. Michael, Topologies on spaces of subsets, Trans. Amer. Math. Soc. (1951), no. 71, $152-182$.

[9] Janusz Pawlikowski, Undetermined sets of point-open games, Fundamenta Mathematicae 144 (1994), no. 3, 279-285.

[10] M. Sakai and M. Scheepers, The combinatorics of open covers, Recent Progress in General Topology III (K.P. Hart, J. van Mill, and P. Simon, eds.), Atlantis Press, 2014, pp. 751-800.

[11] Ratislav Telgársky, Spaces defined by topological games, Fundamenta Mathematicae 88 (1975), no. 3, 193-223.

[12] V. V. Tkachuk, A Cp-Theory Problem Book. Functional Equivalencies, Springer, Cham, 2016.

[13] _ Closed discrete selections for sequences of open sets in function spaces, Acta Mathematica Hungarica 154 (2018), no. 1, 56-68.

[14] — Two point-picking games derived from a property of function spaces, Quaestiones Mathematicae 41 (2018), no. 6, 729-743. 